\title{
Robust Observer-Based Stabilization of Lipschitz Nonlinear Uncertain Systems via LMIs - Discussions and New Design Procedure
}

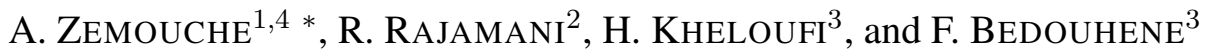 \\ ${ }^{1}$ University of Lorraine, CRAN UMR CNRS 7039, 54400 Cosnes et Romain, France. \\ ${ }^{2}$ Laboratory for Innovations in Sensing, Estimation, and Control, Department of Mechanical Engineering, University of Minnesota, \\ Minneapolis, USA. \\ ${ }^{3}$ Department of Mathematics, Faculty of Sciences, University Mouloud Mammeri, Tizi-Ouzou, Algeria. \\ ${ }^{4}$ EPI Inria DISCO, Laboratoire des Signaux et Systèmes, CNRS-Centrale Supelec, 91192 Gif-sur-Yvette, France.
}

\begin{abstract}
SUMMARY
This paper presents a new observer-based controller design method for Lipschitz nonlinear systems with uncertain parameters and $\mathcal{L}_{2}$ bounded disturbance inputs. In the presence of uncertain parameters, the separation principle is not applicable even in the case of linear time invariant systems. A state of the art review for uncertain linear systems is first presented to describe the shortcomings and conservatism of existing results for this problem. Then a new LMI based design technique is developed to solve the problem for both linear and Lipschitz nonlinear systems. The features of the new technique are the use of a new matrix decomposition, the allowance of additional degrees of freedom in design of the observer and controller feedback gains, the elimination of any need to use equality constraints, the allowance of uncertainty in the input matrix and the encompassing of all previous results under one framework. An extensive portfolio of numerical case studies is presented to illustrate the superiority of the developed design technique to existing results for linear systems from literature, and to illustrate application to Lipschitz nonlinear systems.
\end{abstract}

KEY WORDS: Observer-based control; Linear Matrix Inequalities (LMIs); uncertain systems, $\mathcal{H}_{\infty}$ control, Lipschitz nonlinear systems

\footnotetext{
${ }^{*}$ Correspondence to: Ali Zemouche, University of Lorraine, CRAN UMR CNRS 7039, 54400 Cosnes et Romain, France. Email: ali.zemouche@univ-lorraine.fr
} 


\section{INTRODUCTION}

Uncertain parameters are often encountered in many practical control applications because it is frequently difficult to obtain exact mathematical models. This may be due to environmental noises, data errors, aging of systems, uncertain or slowly varying parameters, etc. Many efforts have been devoted to the robust stability and stabilization of linear systems with parameter uncertainties because the presence of uncertainties may cause instability and bad performances on a controlled system. For a recent literature, we refer the readers to [6], [18], [1], [10], [4]. To provide some reasons for uncertain parameters in linear time invariant models, we can mention the following, which constitute one of the main motivation of this work: (1) variations in parameters with varying environmental conditions (for example, the change in tire-road friction coefficient due to ice or snow on a winter road); (2) variations in parameters over time (for example, the slow and steady change in cornering stiffness of a tire over the life of the tire); (3) variations in parameters due to the nonlinear nature of the model (for example, the presence of hysteresis leads to parameters that can vary continuously between upper and lower bounds depending on both the direction and the range of motion).

In the presence of uncertain parameters, the stabilization problem becomes more difficult and involved. The problem is complicated especially when an observer-based controller is used instead of direct static state feedback. Often, not all of the system states are available by measurement, mainly for economical and/or technological reasons. To overcome this problem an observer is usually added in the control loop, in order to recover the unavailable state information [2], [7], [22], [13], [20]. This is the main motivation for which state observer design for uncertain and/or nonlinear systems is widely investigated in the literature to develop new techniques for different classes of nonlinear systems [5], [27], [28], [29]. For uncertain systems, the separation principle is not applicable even in the linear time invariant case. Trying to ensure that the closed-loop system is stable, while using an observer leads to a gain selection problem difficult from the LMI point of view. There are considerable approaches in the literature dealing with output feedback controller design by directly using BMI conditions [19], [17]. However, it is well known that solving a BMI is an NP-hard problem [25]. To overcome this obstacle, some important and general dynamic output-feedback approaches have been presented in [21] using relevant arguments and judicious mathematical tools. Nevertheless, in this paper we focus our study on observer-based controller design, which presents some difficulties due to its particular structure. Indeed, when 
a Luenberger observer with constant gain is used, the controller design involves the presence of unavoidable bilinear terms, contrarily to the case of dynamic Luenberger observer-based controller [24] or the general dynamic output feedback controller [21], where congruence transformations may be used. On the other hand, static output feedback also has this particularity and leads to an unavoidable BMI, but the use of estimated states provides more degrees of freedom in the controller design and is enable to better estimate the states and the output measurements in the presence of disturbances. Several papers in the literature have addressed the robust stabilization issue via constant gain Luenberger observers for linear systems with parametric uncertainties, but the results remain conservative. We refer the readers to [6], [18], [1], [10], [4], [15], for some recent work in this area.

This paper deals with the problem of observer-based control design via LMIs. Thanks to a new matrix decomposition, we first propose new and enhanced LMI conditions to solve the problem of stabilization of linear systems with uncertain parameters. Indeed, we propose a new two-step procedure, which contains more degrees of design freedom than the classical two-steps algorithm. We show that the proposed method encompasses the Young's relation based approach [15], [26] and Lien's approach [18]. Moreover, the stability of the proposed observer-based scheme is proved also in the presence of Lipschitz nonlinearities and disturbances by resorting to an $\mathcal{H}_{\infty}$ approach according to [27] and [16]. Analytical and simulation comparisons are provided to demonstrate the superiority of the new LMI design methodology compared to previous results.

The present work is divided into 5 sections. The second section provides the statement of the problem and a brief state of the art review related to different LMI design methods available in the literature to examine what already exists in the academic literature. The third section is devoted to the new design procedure, including the presentation of some analytical comparisons. The fourth section reports the main results with Lipschitz nonlinearities and disturbances. Section 5 deals with the presentation of simulation examples and comparisons to show the superiority of the proposed design methodology.Finally, The conclusion at the end sumps up all the work. 


\section{PROBLEM STATEMENT AND BACKGROUND RESULTS}

\subsection{Problem statement}

For the simplicity of the presentation, this part of the study starts by introducing the problem formulation and the new contributions in the linear case without disturbances. Therefore, we consider the same class of systems investigated in [18] and [15], defined by the following set of equations:

$$
\begin{gathered}
\dot{x}=(A+\Delta A(t)) x+B u \\
y=(C+\Delta C(t)) x
\end{gathered}
$$

where $x \in \mathbb{R}^{n}$ is the state vector, $y \in \mathbb{R}^{p}$ is the output measurement and $u \in \mathbb{R}^{m}$ is the control input vector. $A, B$ and $C$ are constant matrices of adequate dimensions. First, we consider the following assumptions :

- the pairs $(A, B)$ and $(A, C)$ are respectively stabilizable and detectable;

- there exist matrices $M_{i}, N_{i}, F_{i}(t), i=1,2$, of appropriate dimensions so that

$$
\Delta A(t)=M_{1} F_{1}(t) N_{1}, \quad \Delta C(t)=M_{2} F_{2}(t) N_{2}
$$

where the unknown matrices $F_{i}(t)$ satisfy the condition

$$
F_{i}^{T}(t) F_{i}(t) \leq I, \text { for } i=1,2
$$

The observer-based controller we consider in this paper is under the form :

$$
\begin{gathered}
\dot{\hat{x}}=A \hat{x}+B u+L(y-C \hat{x}) \\
u=-K \hat{x}
\end{gathered}
$$

where $\hat{x} \in \mathbb{R}^{n}$ is the estimate of $x, K \in \mathbb{R}^{m \times n}$ is the control gain, $L \in \mathbb{R}^{n \times p}$ is the observer gain. Hence, we can write

$$
\left[\begin{array}{l}
\dot{x} \\
\dot{e}
\end{array}\right]=\left[\begin{array}{cc}
(A-B K+\Delta A(t)) & B K \\
(\Delta A(t)-L \Delta C(t)) & (A-L C)
\end{array}\right]\left[\begin{array}{l}
x \\
e
\end{array}\right]
$$

where $e=x-\hat{x}$ represents the estimation error.

We take the same Lyapunov function candidate as in [15],

$$
V(x, e)=\left[\begin{array}{l}
x \\
e
\end{array}\right]^{T}\left[\begin{array}{ll}
P & 0 \\
0 & R
\end{array}\right]\left[\begin{array}{l}
x \\
e
\end{array}\right]=x^{T} P x+e^{T} R e .
$$


Notice that the Lyapunov function (6) is well known in the literature for this problem, especially in [18] which is the main motivation of this paper. Indeed, the main contribution of this paper consists of developing a new design methodology that we compare efficiently to the design methods provided in [18]. Now, after calculating the derivative of $V$ along the trajectories of (5), we have:

$$
\begin{aligned}
\dot{V} \leq & x^{T}\left[(A-B K)^{T} P+P(A-B K)\right] x \\
& +e^{T}\left[(A-L C)^{T} R+R(A-L C)\right] e+2 x^{T} P B K e \\
& +x^{T}\left[\left(\epsilon_{1}+\epsilon_{2}\right) N_{1}^{T} N_{1}+\epsilon_{3} N_{2}^{T} N_{2}+\frac{1}{\epsilon_{1}} P M_{1} M_{1}^{T} P\right] x \\
& +e^{T}\left[\frac{1}{\epsilon_{2}} R M_{1} M_{1}^{T} R+\frac{1}{\epsilon_{3}} R L M_{2} M_{2}^{T} L^{T} R\right] e \\
& =[x]\left[\begin{array}{ll}
\Sigma_{11} & P B K \\
(\star) & \Sigma_{22}
\end{array}\right]\left[\begin{array}{l}
x \\
(\star
\end{array}\right]
\end{aligned}
$$

where

$$
\begin{aligned}
\Sigma_{11}= & {\left[(A-B K)^{T} P+P(A-B K)\right] } \\
+ & {\left[\left(\epsilon_{1}+\epsilon_{2}\right) N_{1}^{T} N_{1}+\epsilon_{3} N_{2}^{T} N_{2}+\frac{1}{\epsilon_{1}} P M_{1} M_{1}^{T} P\right] } \\
\Sigma_{22}= & {\left[(A-L C)^{T} R+R(A-L C)\right] } \\
+ & {\left[\frac{1}{\epsilon_{2}} R M_{1} M_{1}^{T} R+\frac{1}{\epsilon_{3}} R L M_{2} M_{2}^{T} L^{T} R\right] }
\end{aligned}
$$

and $\epsilon_{1}, \epsilon_{2}, \epsilon_{3}$ are positive real constants (for more details, see [18], eq. (7)).

$$
\begin{aligned}
& \text { Notice that } \dot{V}<0, \forall\left[\begin{array}{l}
x \\
e
\end{array}\right] \neq 0 \text { if the matrix inequality } \\
& \qquad\left[\begin{array}{cc}
\Sigma_{11} & P B K \\
(\star) & \Sigma_{22}
\end{array}\right]<0
\end{aligned}
$$

holds. However, the matrix inequality (10) is a Bilinear Matrix Inequality (BMI), which is not exploitable numerically to solve for $P, K$ and $L$. On the other hand, linearizing the BMI (10) is a very difficult task because of the presence of the coupling term $P B K$ (see e.g. [3]). Many researchers in this field have attempted to solve this problem but the resulting methods remain conservative [18], [11], [8], [6], [10]. In the following 
subsection, we recall some available results in the literature and we describe, with details, especially the results of Lien in [18] which constitutes the main motivation of this paper.

\subsection{Background results}

In this subsection, some LMI methods are recalled concerning with this paper. First, the LMI results given in [18] for asymptotic stability of the system (5) are summarized. After this, the LMI design method established in [15] is recalled and slightly improved.

\section{Theorem $2.1([18])$}

System (1a) is asymptotically stabilizable by (4) if there exist some positive constants $\epsilon_{1}, \epsilon_{2}, \epsilon_{3}$, a positive definite matrix $R \in \mathbb{R}^{n \times n}$, and $K \in \mathbb{R}^{m \times n}, \hat{L} \in \mathbb{R}^{n \times p}$ such that

$$
\left[\begin{array}{ccccc}
X_{11} & B K & M_{1} & 0 & 0 \\
(\star) & X_{22} & 0 & R M_{1} & \hat{L} M_{2} \\
(\star) & (\star) & -\epsilon_{1} I & 0 & 0 \\
(\star) & (\star) & (\star) & -\epsilon_{2} I & 0 \\
(\star) & (\star) & (\star) & (\star) & -\epsilon_{3} I
\end{array}\right]<0
$$

where

$$
\begin{gathered}
X_{11}=A^{T}+A-K^{T} B^{T}-B K+\left(\epsilon_{1}+\epsilon_{2}\right) N_{1}^{T} N_{1}+\epsilon_{3} N_{2}^{T} N_{2} \\
X_{22}=A^{T} R+R A-\hat{L} C-C^{T} \hat{L}^{T} .
\end{gathered}
$$

The stabilizing observer-based control gains are given by $K$ and $L=R^{-1} \hat{L}$.

In the proof of this theorem, the author made the particular choice of $P$, namely $P=I$, in order to linearize the BMI (10). Faced with this strong restriction, the author introduced a new matrix $\hat{P}$ satisfying the condition $P B=\hat{P} B$. Such a matrix is necessarily invertible. By putting $\hat{K}=\hat{P} K$ in (10), he obtained the following synthesis conditions :

Theorem $2.2([18])$

System (1a) is asymptotically stabilizable by (4) if there exist some positive constants $\epsilon_{1}, \epsilon_{2}, \epsilon_{3}$, two positive 
definite matrices $P, R \in \mathbb{R}^{n \times n}$, and matrices $\hat{K} \in \mathbb{R}^{m \times n}, \hat{L} \in \mathbb{R}^{n \times p}, \hat{P} \in \mathbb{R}^{m \times m}$ such that

$$
\left[\begin{array}{ccccc}
Y_{11} & B \hat{K} & P M_{1} & 0 & 0 \\
(\star) & Y_{22} & 0 & R M_{1} & \hat{L} M_{2} \\
(\star) & (\star) & -\epsilon_{1} I & 0 & 0 \\
(\star) & (\star) & (\star) & -\epsilon_{2} I & 0 \\
(\star) & (\star) & (\star) & (\star) & -\epsilon_{3} I
\end{array}\right]<0
$$

where

$$
\begin{gathered}
Y_{11}=A^{T} P+P A-\hat{K}^{T} B^{T}-B \hat{K}+\left(\epsilon_{1}+\epsilon_{2}\right) N_{1}^{T} N_{1}+\epsilon_{3} N_{2}^{T} N_{2} \\
Y_{22}=A^{T} R+R A-\hat{L} C-C^{T} \hat{L}^{T} .
\end{gathered}
$$

The stabilizing observer-based control gains are given by $K=\hat{P}^{-1} \hat{K}$ and $L=R^{-1} \hat{L}$.

Notice that even if Theorem 2.2 provides less restrictive synthesis conditions than that of Theorem 2.1, it remains quite conservative because of the presence of the equality constraint (13).

In the next theorem, we recall the new design methodology presented firstly in [14]. Notice that this theorem can be found also in [26] as a corrected version of [15]. This method proposes a novel manner to overcome the obstacle of the coupling $P B K$ without any equality constraint.

Theorem 2.3 ([26])

System (1a) is asymptotically stabilizable by (4) if for fixed scalars $\epsilon_{2}>0, \epsilon_{3}>0$ and $\epsilon_{4}>0$, there exist two positive definite matrices $\mathcal{Z} \in \mathbb{R}^{n \times n}, R \in \mathbb{R}^{n \times n}$, two matrices $\hat{K} \in \mathbb{R}^{n \times m}, \hat{L} \in \mathbb{R}^{p \times n}$, and a scalar $\epsilon_{1}>0$ so that the following LMI condition (14) is feasible:

$$
\left[\begin{array}{cc}
\mathcal{H}_{11} & \mathcal{H}_{12} \\
(\star) & -\operatorname{diag}\left\{\frac{1}{\epsilon_{4}} \mathcal{Z}, \epsilon_{4} \mathcal{Z}, \epsilon_{1} \mathrm{I}, \epsilon_{2} \mathrm{I}, \frac{1}{\epsilon_{2}}, \epsilon_{3} \mathrm{I}, \frac{1}{\epsilon_{3}} \mathrm{I}\right\}
\end{array}\right]<0
$$

with

$$
\mathcal{H}_{11}=\operatorname{diag}\left\{\operatorname{He}\left(\mathcal{Z} \mathrm{A}^{\mathrm{T}}-\hat{\mathrm{K}} \mathrm{B}^{\mathrm{T}}\right)+\epsilon_{1} \mathrm{M}_{1} \mathrm{M}_{1}^{\mathrm{T}}, \operatorname{He}\left(\mathrm{A}^{\mathrm{T}} \mathrm{R}-\mathrm{C}^{\mathrm{T}} \hat{\mathrm{L}}\right)\right\}
$$




$$
\mathcal{H}_{12}=\left[\begin{array}{ccccccc}
B \hat{K}^{T} & 0 & \mathcal{Z} N_{1}^{T} & 0 & \mathcal{Z} N_{1}^{T} & 0 & \mathcal{Z} N_{2}^{T} \\
& & & & & & \\
0 & I & 0 & R M_{1} & 0 & \hat{L}^{T} M_{2} & 0
\end{array}\right]
$$

where $\operatorname{He}(\mathbb{M})=\mathbb{M}+\mathbb{M}^{T}$ for all square matrix $\mathbb{M}$. Hence, the stabilizing observer-based control gains are given by $K=\hat{K}^{T} \mathcal{Z}^{-1}$ and $L=R^{-1} \hat{L}^{T}$.

Notice that the scalar variables $\epsilon_{i}, i=2,3,4$ are to be fixed a priori to render linear the condition (14). Moreover, in the goal to overcome this difficulty and to obtain maximum values of the uncertainty bounds tolerated by (14), the bounds of the uncertainties are included in (3), which is replaced by the following:

$$
F_{i}^{T}(t) F_{i}(t) \leq \alpha_{i}^{2} I
$$

This formulation is often used in decentralized stabilization problem of interconnected systems. The objective consists in optimizing the bounds of the uncertainties. Consequently, this idea leads to an LMI without a priori choice of the scalars $\epsilon_{i}, i=1,2,3$.

Under these new considerations, the inequality (7) becomes:

$$
\begin{aligned}
\dot{V} \leq & x^{T}\left[(A-B K)^{T} P+P(A-B K)\right] x \\
+\varepsilon^{T} & {\left[(A-L C)^{T} R+R(A-L C)\right] \varepsilon+2 x^{T} P B K \varepsilon } \\
+x^{T} & {\left[\alpha_{1}^{2}\left(\epsilon_{1}+\epsilon_{2}\right) N_{1}^{T} N_{1}+\epsilon_{3} \alpha_{2}^{2} N_{2}^{T} N_{2}\right.} \\
& \left.+\frac{1}{\epsilon_{1}} P M_{1} M_{1}^{T} P\right] x \\
+\varepsilon^{T} & {\left[\frac{1}{\epsilon_{2}} R M_{1} M_{1}^{T} R+\frac{1}{\epsilon_{3}} R L M_{2} M_{2}^{T} L^{T} R\right] \varepsilon . }
\end{aligned}
$$

Hence, with the change of variables $\gamma_{1}=\frac{1}{\alpha_{1}^{2}\left(\epsilon_{1}+\epsilon_{2}\right)}$ and $\gamma_{2}=\frac{1}{\epsilon_{3} \alpha_{2}^{2}}$, we obtain the following enhanced version of Theorem 2.3.

\section{Theorem 2.4}

Assume that for a fixed scalar $\epsilon_{4}>0$, there exist two positive definite matrices $\mathcal{Z} \in \mathbb{R}^{n \times n}, R \in \mathbb{R}^{n \times n}$, two matrices $\hat{K} \in \mathbb{R}^{n \times m}, \hat{L} \in \mathbb{R}^{p \times n}$ and positive scalars $\epsilon_{i}, i=1,2,3, \gamma_{i}, i=1,2$ so that the following convex optimization problem holds: 


$$
\begin{gathered}
\min \left(\sum_{i=1}^{2} \gamma_{i}+\sum_{i=1}^{3} \epsilon_{i}\right) \text { subject to } \\
\left.\qquad \begin{array}{cccc}
\mathcal{H}_{11} & \mathcal{H}_{12} \\
(\star) & -\operatorname{diag}\left\{\frac{1}{\epsilon_{4}} \mathcal{Z}, \epsilon_{4} \mathcal{Z}, \epsilon_{1} \mathrm{I}, \epsilon_{2} \mathrm{I}, \gamma_{1} \mathrm{I}, \epsilon_{3} \mathrm{I}, \gamma_{2} \mathrm{I}\right\}
\end{array}\right]<0 \\
\mathcal{H}_{11}=\operatorname{diag}\left\{\operatorname{He}\left(\mathcal{Z} \mathrm{A}^{\mathrm{T}}-\hat{\mathrm{K} B}{ }^{\mathrm{T}}\right), \operatorname{He}\left(\mathrm{A}^{\mathrm{T}} \mathrm{R}-\mathrm{C}^{\mathrm{T}} \hat{\mathrm{L}}\right)\right\} \\
\mathcal{H}_{12}=\left[\begin{array}{ccccccc}
B \hat{K}^{T} & 0 & M_{1} & 0 & \mathcal{Z} N_{1}^{T} & 0 & \mathcal{Z} N_{2}^{T} \\
0 & I & 0 & R M_{1} & 0 & \hat{L}^{T} M_{2} & 0
\end{array}\right] .
\end{gathered}
$$

Then, system (1) is asymptotically stabilizable by the observer-based controller gains $K=\hat{K}^{T} \mathcal{Z}$ and $L=$ $R^{-1} \hat{L}^{T}$, for all

$$
\alpha_{1} \leq \frac{1}{\sqrt{\gamma_{1}\left(\epsilon_{1}+\epsilon_{2}\right)}} \text { and } \alpha_{2} \leq \frac{1}{\sqrt{\gamma_{2} \epsilon_{3}}} .
$$

Consequently, it remains only to fix the scalar $\epsilon_{4}$ a priori in (19). To this end, the best manner is the use of the gridding method as suggested in [15] and [14]. A linearized version of Theorem 2.4 with respect to $\epsilon_{4}$ can be obtained by using the additional constraint

$$
\mathcal{Z}>\alpha I
$$

This allows us to get an LMI on $\alpha$ and $\beta=\alpha \epsilon_{4}$, instead of the BMI (19) on $\epsilon_{4}$ (see [15, Remark 2, Eq. (13)] for more details). Notice also that if $\alpha_{1}$ and $\alpha_{2}$ are fixed a priori, we get Theorem 2.3 from Theorem 2.4. We need only some Schur arrangements in (18).

\section{ENHANCED LMI DESIGN PROCEDURE}

This section is devoted to a new enhanced LMI design algorithm. First, we introduce a novel LMI condition, which is more general and less conservative than that in [18]. This new design procedure is able to solve the observer-based stabilization problem for a large class of uncertain systems. We improve the approach in [18] by presenting a general LMI condition, where the conditions in [18] become a particular case of the general one. Inspired by the classical two-steps algorithms for observer-based controllers design [23], a new two-steps algorithm is presented with more degrees of freedom than the classical technique. 


\subsection{Preliminaries}

Since $B$ is full column rank, there always exists a non singular matrix $T$ so that $T B=\left[\begin{array}{c}I_{m} \\ 0\end{array}\right]$. Hence, using the similarity transformation $T$, system (1) can be transformed under the following equivalent form:

$$
\begin{gathered}
A:=T A T^{-1}, C:=C T^{-1}, B:=T B=\left[\begin{array}{c}
I_{m} \\
0
\end{array}\right], \\
\Delta A(t):=T \Delta A(t) T^{-1}, \Delta C(t):=\Delta C(t) T^{-1}, \\
M_{1}:=T M_{1}, N_{1}:=N_{1} T^{-1}, M_{2}:=M_{2}, N_{2}:=N_{2} T^{-1} .
\end{gathered}
$$

Thanks to these assignments, the redundancy is avoided, and then we obtain the same structure (1) with

$$
B=\left[\begin{array}{c}
I_{m} \\
0
\end{array}\right]
$$

Now, the matrices $P$ and $A$ are written in the detailed forms as follows:

$$
A=\left[\begin{array}{cc}
A_{11} & A_{12} \\
A_{21} & A_{22}
\end{array}\right], P=\left[\begin{array}{cc}
P_{11} & P_{12} \\
P_{12}^{T} & P_{22}
\end{array}\right] .
$$

Since what we propose is a generalization of the methodology in [18], let us starting from inequality (10). By taking into account the structure (22) of $B$, we get

$$
P B=\left[\begin{array}{cc}
P_{11} & P_{12} \\
P_{12}^{T} & P_{22}
\end{array}\right]\left[\begin{array}{c}
I_{m} \\
0
\end{array}\right]=\left[\begin{array}{c}
P_{11} \\
P_{12}^{T}
\end{array}\right]
$$

and therefore

$$
P B K=\left[\begin{array}{c}
P_{11} K \\
P_{12}^{T} K
\end{array}\right] .
$$

Hence, from (24), equality (8) becomes:

$$
\begin{aligned}
\Sigma_{11}= & {\left[A^{T} P+P A-\left[\begin{array}{c}
P_{11} K \\
P_{12}^{T} K
\end{array}\right]-\left[\begin{array}{c}
P_{11} K \\
P_{12}^{T} K
\end{array}\right]^{T}\right] } \\
& +\left[\left(\epsilon_{1}+\epsilon_{2}\right) N_{1}^{T} N_{1}+\epsilon_{3} N_{2}^{T} N_{2}+\frac{1}{\epsilon_{1}} P M_{1} M_{1}^{T} P\right] .
\end{aligned}
$$




\section{Remark 3.1}

The transformation of the $B$ matrix as in (22) is well known in the literature for the problem of static output feedback. The novelty of our methodology lies in the manner to exploit this transformation to extract a part of the Lyapunov matrix instead of the controller gain as usually used in the two-steps method. Thanks to this technique, the observer-based controller gains will be computed simultaneously after solving an LMI having more degrees of freedom than the standard two-steps algorithm (see subsection 3.3.3).

\subsection{New design procedure}

Since the matrix $P_{11}$ is symmetric positive definite, then invertible, there always exists a matrix $S \in \mathbb{R}^{m \times(n-m)}$ so that

$$
P_{12}=P_{11} S
$$

With the change of variables $\hat{K}=P_{11} K, \hat{L}=R L$, and the use of Schur Lemma, we deduce that inequality (10) is fulfilled if the following matrix inequality holds:

$$
\left[\begin{array}{ccccc}
\Gamma_{11} & {\left[\begin{array}{c}
\hat{K} \\
S^{T} \hat{K}
\end{array}\right]} & P M_{1} & 0 & 0 \\
(\star) & \Gamma_{22} & 0 & R M_{1} & \hat{L} M_{2} \\
(\star) & (\star) & -\epsilon_{1} I & 0 & 0 \\
(\star) & (\star) & (\star) & -\epsilon_{2} I & 0 \\
(\star) & (\star) & (\star) & (\star) & -\epsilon_{3} I
\end{array}\right]<0
$$

where

$$
\begin{aligned}
\Gamma_{11}= & A^{T} P+P A-\left[\begin{array}{c}
\hat{K} \\
S^{T} \hat{K}
\end{array}\right]^{T}-\left[\begin{array}{c}
\hat{K} \\
S^{T} \hat{K}
\end{array}\right] \\
& +\left(\epsilon_{1}+\epsilon_{2}\right) N_{1}^{T} N_{1}+\epsilon_{3} N_{2}^{T} N_{2} \\
\Gamma_{22}= & A^{T} R+R A-\hat{L} C-C^{T} \hat{L}^{T} .
\end{aligned}
$$


The matrix inequality (27) is not linear because of the presence of the coupling term $S^{T} \hat{K}$. To linearize it, the two-steps design is suggested, but with a different manner than the classical two-steps algorithm where the controller gain is computed a priori and considered as known in (27). The proposed procedure is as follows:

- put $S=\alpha \bar{S}$ and compute the matrix $\bar{S}$ as a solution of the LMI resulting from the stabilization of (1a) by a static state feedback $u=-\mathcal{K} x$

- solve (27) with $S=\alpha \bar{S}$ by using the gridding method on $\alpha$ with the decision variables $P_{11}, P_{22}, R, \hat{K}, \hat{L}$.

\section{Remark 3.2}

Notice that the form $\bar{S}=P_{11}^{-1} P_{12}$ is not a restriction. This form, always true and possible [3], is introduced and used in the goal to retrieve the variable $\hat{K} \cdot \bar{S}$ is introduced in the aim to include an additional degree of freedom in the algorithm, namely $\alpha$, (or $\Lambda$ in subsection 3.4 , for more relaxed version), contrarily to the classical two-step algorithm where the control gain $K$ is fixed in the second step without any additional degree of freedom to improve compensation of the first step.

Now, return to the computation of the matrix $\bar{S}$. With a static state feedback $u=-\mathcal{K} x$, system (1a) can be written in the closed-loop form:

$$
\dot{x}=\left(A-B \mathcal{K}+M_{1} F_{1}(t) N_{1}\right) x
$$

System (30) is asymptotically stabilized by the Lyapunov function $V(x)=x^{T} \mathcal{P} x$ if the following inequality holds:

$$
(A-B \mathcal{K})^{T} \mathcal{P}+\mathcal{P}(A-B \mathcal{K})+N_{1}^{T} F_{1}^{T}(t) M_{1}^{T} \mathcal{P}+\mathcal{P} M_{1} F_{1}(t) N_{1}<0
$$

Using, on (31), the congruence principle, the Young inequality with (3) and Schur Lemma, we conclude that (30) is asymptotically stable if the following LMI condition is fulfilled:

$$
\left[\begin{array}{cc}
\mathcal{Z} A^{T}+A \mathcal{Z}-\overline{\mathcal{K}}^{T} B^{T}-B \overline{\mathcal{K}}+\epsilon M_{1} M_{1}^{T} & \mathcal{Z} N_{1}^{T} \\
N_{1} \mathcal{Z} & -\epsilon I_{n}
\end{array}\right]<0
$$

where $\overline{\mathcal{K}}=\mathcal{K} \mathcal{Z}$ and $\mathcal{Z}^{-1}=\mathcal{P}=\left[\begin{array}{cc}\mathcal{P}_{11} & \mathcal{P}_{12} \\ \mathcal{P}_{12}^{T} & \mathcal{P}_{22}\end{array}\right]$. The classical two-steps method is different from our new design technique. Indeed, the standard method proceeds as in the following algorithm: 


\section{Algorithm 1: (classical two-steps method)}

1. Solve LMI (32) with the decision variables $\mathcal{Z}, \overline{\mathcal{K}}$ and $\epsilon>0$;

2. compute $\mathcal{K}$ by $\mathcal{K}=\mathcal{P}_{11}^{-1} \overline{\mathcal{K}}$;

3. solve (27) for $K=\mathcal{K}$ with the decision variables $P_{11}, P_{12}, P_{22}, R, \hat{L}$ and $\epsilon_{i}, i=1,2,3$;

4. compute the observer gain as $L=R^{-1} \hat{L}$.

Nevertheless, our design algorithm works in a different manner, as summarized in the following:

\section{$\underline{\text { Algorithm } 2}$}

1. Solve LMI (32) with the decision variables $\mathcal{Z}, \overline{\mathcal{K}}$ and $\epsilon>0$;

2. compute $\bar{S}$ by $\bar{S}=\mathcal{P}_{11}^{-1} \mathcal{P}_{12}$, and put $S=\alpha \bar{S}$;

3. solve (27) with $S=\alpha \bar{S}$ by using the gridding method on $\alpha$ with the decision variables $P_{11}, P_{22}, R, \hat{K}, \hat{L}$ and $\epsilon_{i}, i=1,2,3$;

4. compute the observer-based controller gains as $K=P_{11}^{-1} \hat{K}$ and $L=R^{-1} \hat{L}$.

\subsection{Some remarks and comparisons}

The first step consists in fixing the structure of the matrix $S$ by $\bar{S}$. Then, the parameter $\alpha$ is introduced to add a degree of freedom for (27) and then relaxing it. To choose $\alpha$, the gridding method is used. Indeed, the gridding method in this case, consists in writing $\alpha=\frac{\kappa}{1-\kappa^{2}}$, which means that

$$
\kappa \in]-1,1[\Longleftrightarrow \alpha \in]-\infty,+\infty[
$$

In fact, the function:

$$
\begin{array}{r}
]-1,1[\longrightarrow]-\infty,+\infty[ \\
\kappa \longmapsto \alpha(\kappa)=\frac{\kappa}{1-\kappa^{2}}
\end{array}
$$


is bijective (a monotone strictly increasing function) (a $\mathcal{C}^{1}$-diffeomorphism to be more precise). In addition, its inverse function is given by

$$
\kappa(\alpha)=\left\{\begin{array}{ccc}
\frac{1}{2 \alpha}\left(\sqrt{4 \alpha^{2}+1}-1\right) & \text { if } & \alpha>0 \\
-\frac{1}{2 \alpha}\left(\sqrt{4 \alpha^{2}+1}-1\right) & \text { if } & \alpha<0 \\
0 & \text { if } & \alpha=0 .
\end{array}\right.
$$

Then, we assign a uniform subdivision of the interval $]-1,1[$ and we solve the LMI (27) for each value of this subdivision.

3.3.1. Comparison to Lien's method It is evident that the approach in [18] becomes a particular case of this new procedure. Indeed, first, if LMI (11) is assumed feasible, then it is clear that the proposed LMI (27) is also feasible for $P_{11}=I_{m}, P_{22}=I_{n-m}$ and $\alpha=0$. Now, assume that (12)-(13) are verified. Then, from (13) is derived the following:

$$
P B=B \hat{P} \Longrightarrow\left[\begin{array}{l}
P_{11} \\
P_{12}^{T}
\end{array}\right]=\left[\begin{array}{l}
\hat{P} \\
0
\end{array}\right]
$$

which means that $P_{11}=\hat{P}$ and $P_{12}=0$. Consequently, LMI (27) is also satisfied because it is identical to (12) for $P_{11}=\hat{P}$ and $\alpha=0$. It should be noticed that in the case of LMI (11) or (12)-(13), the first step of the new proposed LMI design algorithm is not required. The algorithm is reduced to one step, namely the step (3) (LMI (27) with $S=0$ ). On the other hand, the converse is not true. That is, if the new design algorithm provides solutions, then the LMIs (11) and (12)-(13), do not provides automatically solutions. The following section presents numerical examples, which show that the proposed design method provides less conservative LMI conditions.

We point out that the approach in [18] is constrained by the Schur stability of the matrix block $A_{22}$ of $A$. In fact, if $P_{12}=0$, which corresponds to $S=0$ in (27), we deduce from (27) and (28) that

$$
A_{22}^{T} P_{22}+P_{22} A_{22}<0
$$

On the other hand, notice that this necessary condition is not needed with this new design procedure, because the matrix $S$ is not necessarily null.

In addition, it should be noticed that the equality constraint (13) is more strong than the Schur stability of $A_{22}$. Indeed, as demonstrated in [15, subsection 3.2], all the diagonal sub-blocks of $A_{22}$ should be Schur stable. 
Indeed, let

$$
\begin{aligned}
& A_{22}^{i} \in \mathbb{R}^{(n-m-i) \times(n-m-i)}, i=0, \ldots, n-m \\
& P_{22}^{i} \in \mathbb{R}^{(n-m-i) \times(n-m-i)}, i=0, \ldots, n-m
\end{aligned}
$$

be the diagonal sub-blocks of $A_{22}$ and $P_{22}$, respectively, with $A_{22}^{0}=A_{22}$ and $P_{22}^{0}=P_{22}$. Then, if the equality constraint (13) is satisfied conjointly with (12), then it is necessary to have

$$
A_{22}^{i^{T}} P_{22}^{i}+P_{22}^{i} A_{22}^{i}<0, \forall i=0, \ldots n-m
$$

To prove (35), the following notations are introduced for all $i=0, \ldots, n-m$ :

$$
\begin{gathered}
B^{i}=\left[\begin{array}{c}
I_{m} \\
0 \\
\vdots \\
\underbrace{0}_{i^{t h} \text { zero }}
\end{array}\right], B^{0}=I_{m}, \\
A=\left[\begin{array}{ll}
A_{11}^{i} & A_{12}^{i} \\
A_{21}^{i} & A_{22}^{i}
\end{array}\right], P=\left[\begin{array}{cc}
P_{11}^{i} & P_{12}^{i} \\
\left(P_{12}^{i}\right)^{T} & P_{22}^{i}
\end{array}\right] .
\end{gathered}
$$

Hence, from the equality constraint (13) it results

$$
B^{\perp} P B=B^{\perp} B \hat{P}=\left(P_{12}^{i}\right)^{T}=0, \forall i=1, \ldots, n-m
$$

where

$$
B^{\perp}=\left[\begin{array}{ll}
0_{m+i} & I_{n-m-i}
\end{array}\right]
$$

Following the results in [15, subsection 3.2], it is straightforward to show that (35) is necessary for the feasibility of (12).

To sum up, the fact that $P_{12}^{i}=0, \forall i=1, \ldots, n-m$ means that $P_{22}$ defined in (34) is diagonal. This implies that the equality constraint is more conservative than the condition $P_{12}=0$.

3.3.2. About the Young's relation based approach The difference between this new design methodology and that based on a judicious use of Young's inequality [15], is that the proposed approach does not need to use any strong inequality. Indeed, the approach in [15] is useful and works successfully, but because of the uncertainties 
it may fail for some systems, as can be shown in [26]. Consequently, as a conclusion, these two methods are alternative, but from the feasibility point of view, both give the same results in the parameter uncertainty-free case. In the presence of uncertainties in the state and output equations, the proposed new design is often much more interesting. Analytically, it is difficult to show the superiority of the new design algorithm. However, both the methods are considered alternative methods.

3.3.3. About the classical two-steps method The first advantage of our design method compared to the classical two-steps technique is that the controller gain is not fixed a priori from (32). We have only to a priori fixe the structure of $\bar{S}$ from (32). Hence, the observer and controller gains are computed simultaneously after solving (27). This technique allows to have more degree of freedom compared to the classical method. Indeed, the classical two-steps algorithm consists of fixing $n \times m$ variables, while with the proposed new algorithm, only $(n-m) \times m$ variables are fixed a priori. With the additional parameter $\alpha$, which is introduced to enhance the feasibility of (27), the new design algorithm contains $m^{2}+1$ additional degrees of freedom.

\subsection{Relaxed LMI design}

As can be shown below, the proposed new algorithm has some advantages compared to the previous ones. The introduction of the parameter $\alpha$ increases the degree of freedom and the chance to find solutions for (27). Nevertheless, the proposed approach can be improved by replacing $\alpha$ by a diagonal multiplier matrix

$$
\Lambda=\operatorname{diag}\left(\alpha_{1}, \ldots, \alpha_{\mathrm{m}}\right)
$$

Hence, our algorithm can be reformulated as follows:

\section{Algorithm 3}

1. Solve LMI (32) with the decision variables $\mathcal{Z}, \overline{\mathcal{K}}$ and $\epsilon>0$;

2. compute $\bar{S}$ by $\bar{S}=\mathcal{P}_{11}^{-1} \mathcal{P}_{12}$, and put $S=\Lambda \bar{S}$;

3. solve (27) with $S=\Lambda \bar{S}$ by using the gridding method on $\alpha_{i}, i=1, \ldots, m$, with the decision variables

$$
P_{11}, P_{22}, R, \hat{K}, \hat{L} \text { and } \epsilon_{i}, i=1,2,3 \text {; }
$$

4. compute the observer-based controller gains as $K=P_{11}^{-1} \hat{K}$ and $L=R^{-1} \hat{L}$. 
In this case, compared to the standard two-steps algorithm, this relaxed design contains $m(m+1)$ additional degree of freedom. Now, as mentioned above in subsection 3.3.2, this new algorithm and the Young's relation based approach may be viewed as two alternative methods. Indeed, analytically speaking, we cannot compare between these two methods. The advantage of the first one is that it works in one step, while the second one works on two-steps. Combining these two methods, we propose the following algorithm, which is the general new procedure.

\section{Algorithm 4: general algorithm}

step 1: Solve LMI (14) by using the gridding method on $\epsilon_{i}, i=1,2,3,4$, with the decision variables $\mathcal{Z}, R, \hat{K}$ and $\hat{L}$, and go to step 2 ;

step 2: If LMI (14) is found feasible, then compute the observer-based controller gains as $K=\hat{K}^{T} \mathcal{Z}^{-1}$ and $L=R^{-1} \hat{L}^{T}$. Otherwise, go to step 3;

step 3: Solve LMI (32) with the decision variables $\mathcal{Z}, \overline{\mathcal{K}}$ and $\epsilon>0$ and go to step 4;

step 4: compute $\bar{S}$ by $\bar{S}=\mathcal{P}_{11}^{-1} \mathcal{P}_{12}$, put $S=\Lambda \bar{S}$ and go to step 5;

step 5: Solve (27) with $S=\Lambda \bar{S}$ by using the gridding method on $\alpha_{i}, i=1, \ldots, m$, with the decision variables $P_{11}, P_{22}, R, \hat{K}, \hat{L}$ and $\epsilon_{i}, i=1,2,3$, and go to step 6 ;

step 6 Compute the observer-based controller gains as $K=P_{11}^{-1} \hat{K}$ and $L=R^{-1} \hat{L}$.

Notice that this complete algorithm encompasses Lien's methods and the Young inequality based approach. In fact, we have proposed to include the Young relation based approach in this algorithm because it works in only one step and then in the case where it provides solutions, it would be not necessary to solve Algorithm 3.

\subsection{Case of Uncertainty in the Input Matrix}

In this subsection, we will extend our design method by addressing uncertainty in the input matrix $B$. Consider systems under the following form:

$$
\begin{gathered}
\dot{x}=(A+\Delta A(t)) x+(B+\Delta B(t)) u \\
y=(C+\Delta C(t)) x
\end{gathered}
$$


where $\Delta B(t)=M_{3} F_{3}(t) N_{3}$, with $F_{3}$ satisfying inequality (3).

From seeing the coordinate transformation $T$ defined in subsection 3.1, it is clear that allowing uncertainty in the matrix $B$ is non-trivial. Hence, the following extension of the state vector is proposed to accommodate uncertainty in $B$.

3.5.1. Additional integrator: Introduce a new state modified control input, $w$. Let this state be related to the original control input $u$ by the following equation:

$$
\tau \dot{u}+u=w .
$$

Equation (37) is just a relationship in which the bandwidth of the actuator is assumed to be $\frac{1}{\tau}$.

With an extended state space vector, the new plant equations can be written so as to have no uncertainty in the new $B$ matrix:

$$
\begin{gathered}
\dot{\xi}=(\mathbb{A}+\Delta \mathbb{A}) \xi+\mathbb{B} u_{\xi} \\
y=(\mathbb{C}+\Delta \mathbb{C}) \xi
\end{gathered}
$$

where

$$
\begin{gathered}
\mathbb{A} \triangleq\left[\begin{array}{cc}
-\frac{1}{\tau} I_{m} & 0_{m \times n} \\
B & A
\end{array}\right], \mathbb{B} \triangleq\left[\begin{array}{c}
I_{m} \\
0_{n \times m}
\end{array}\right], \mathbb{C} \triangleq\left[\begin{array}{cc}
0_{p \times m} & C
\end{array}\right], \\
\xi \triangleq\left[\begin{array}{l}
u \\
x
\end{array}\right], u_{\xi}=\frac{1}{\tau} w
\end{gathered}
$$

and

$$
\Delta \mathbb{A} \triangleq \mathbb{M}_{1} \mathbb{F}_{1} \mathbb{N}_{1}, \Delta \mathbb{C} \triangleq \mathbb{M}_{2} \mathbb{F}_{2} \mathbb{N}_{2}
$$

with

$$
\begin{gathered}
\mathbb{M}_{1}=\left[\begin{array}{cc}
0 & 0 \\
M_{3} & M_{1}
\end{array}\right], \mathbb{N}_{1}=\left[\begin{array}{cc}
N_{3} & 0 \\
0 & N_{1}
\end{array}\right], \\
\mathbb{M}_{2}=M_{2}, \mathbb{N}_{2}=\left[\begin{array}{ll}
0_{p \times m} & N_{2}
\end{array}\right], \\
\mathbb{F}_{1}=\left[\begin{array}{cc}
F_{3} & 0 \\
0 & F_{1}
\end{array}\right], \mathbb{F}_{2}=F_{2} .
\end{gathered}
$$


It is clear that $\mathbb{F}_{1}$ and $\mathbb{F}_{2}$ satisfy inequality (3).

Note that the new $\mathbb{B}$ matrix only involves the parameter $\tau$, which can be assumed to have no uncertainty, since it is just the bandwidth of the actuator.

Now, we consider the observer-based controller:

$$
\begin{gathered}
\dot{\hat{\xi}}=\mathbb{A} \hat{\xi}+\mathbb{B} u_{\xi}+\mathbb{L}(y-\mathbb{C} \hat{\xi}) \\
u_{\xi}=-\mathbb{K} \hat{\xi}
\end{gathered}
$$

where $\hat{\xi}$ is the estimate of $\xi$ and $\mathbb{L}, \mathbb{K}$ are the observer-based controller gains to be determined so that the estimation error

$$
e_{\xi}=\xi-\hat{\xi}
$$

and $\xi$ converge asymptotically towards zero. Hence, we have

$$
\left[\begin{array}{c}
\dot{\xi} \\
\dot{e}_{\xi}
\end{array}\right]=\left[\begin{array}{cc}
(\mathbb{A}-\mathbb{B} \mathbb{K}+\Delta \mathbb{A}) & \mathbb{B} \mathbb{K} \\
(\Delta \mathbb{A}-\mathbb{L} \Delta \mathbb{C}) & (\mathbb{A}-\mathbb{L} \mathbb{C})
\end{array}\right]\left[\begin{array}{c}
\xi \\
e_{\xi}
\end{array}\right]
$$

Since (57) is exactly in the same form than (5) and the $\mathbb{B}$ matrix is under the form (22), then Algorithm 4 can be applied to deduce the observer-based controller gains $\mathbb{K}$ and $\mathbb{L}$.

According to what we speculated in the previous section, the Lien's approach requiring the equality constraint (13) can return solutions if $\mathbb{A}_{22}=A$ and all its diagonal sub-blocks are Schur stable. Obviously, this strong constraint is very conservative and the drawback is that it appears in most real models and physical applications. Nevertheless, this constraint vanishes with the application of our design methodology.

\section{Remark 3.3}

The control input $u(t)$ will be obtained from equation (37), using the following solution to the equation:

$$
u(t)=\int_{0}^{t} w(t) g(t-\tau) \mathrm{d} \tau
$$

where $g(t)$ is the inverse Laplace transform of the transfer function $G(s)=\frac{1}{\tau s+1}$.

3.5.2. On the stabilizability and detectability of $(\mathbb{A}, \mathbb{B})$ and $(\mathbb{A}, \mathbb{C})$ : Both the stabilizability and detectability conditions on $(\mathbb{A}, \mathbb{B})$ and $(\mathbb{A}, \mathbb{C})$ are preserved under the stabilizability and detectability of $(A, B)$ and $(A, C)$, 
respectively. Notice by $\mathfrak{S} \mathfrak{t a}$ and $\mathfrak{D e t}$, the stabilizability and detectability matrices, respectively, defined by:

$$
\mathfrak{S t a}=\left(\begin{array}{ll}
s \mathbb{I}_{n+m}-\mathbb{A} & \mathbb{B}
\end{array}\right), \quad \mathfrak{D} \mathfrak{e t}=\left(\begin{array}{c}
s \mathbb{I}_{n+m}-\mathbb{A} \\
\mathbb{C}
\end{array}\right),
$$

where $s$ is a complex number. We say that the pair

- $(\mathbb{A}, \mathbb{B})$ is stabilizable iff

$$
\operatorname{rank}(\mathfrak{S t a})=\mathrm{n}+\mathrm{m}, \forall \mathrm{s}, \mathfrak{R e}(\mathrm{s}) \geq 0
$$

- $(\mathbb{A}, \mathbb{C})$ is detectable iff

$$
\operatorname{rank}(\mathfrak{D e t})=\mathrm{n}+\mathrm{m}, \forall \mathrm{s}, \mathfrak{R e}(\mathrm{s}) \geq 0
$$

On the other hand, we have

$$
\begin{gathered}
\operatorname{rank}(\mathfrak{S t a})=\operatorname{rank}\left(\left[\begin{array}{ccc}
\left(s+\frac{1}{\tau}\right) \mathbb{I}_{m} & 0 & \mathbb{I}_{m} \\
-B & s \mathbb{I}_{n}-A & 0_{n \times m}
\end{array}\right]\right), \\
\operatorname{rank}(\mathfrak{D} \mathfrak{t})=\operatorname{rank}\left(\left[\begin{array}{cc}
\left(s+\frac{1}{\tau}\right) \mathbb{I}_{m} & 0 \\
-B & s \mathbb{I}_{n}-A \\
0 & C
\end{array}\right]\right)
\end{gathered}
$$

It is easy to show that

$$
\operatorname{rank}(\mathfrak{S t a})=\operatorname{rank}(\mathfrak{D e t})=\mathrm{n}+\mathrm{m}, \forall \mathrm{s}, \mathfrak{R e}(\mathrm{s}) \geq 0
$$

Indeed, it is clear that

$$
\operatorname{rank}(\mathfrak{S t a})=\overbrace{\operatorname{rank}\left(\left[\begin{array}{ll}
-B & s \mathbb{I}_{n}-A
\end{array}\right]\right)}^{=n:(A, B)}+\operatorname{rank}\left(\mathbb{I}_{m}\right)=n+m
$$

and since $\tau>0$ :

$$
\operatorname{rank}(\mathfrak{D} \mathfrak{e t})=\overbrace{\operatorname{rank}\left(\left[\begin{array}{c}
s \mathbb{I}_{n}-A \\
C
\end{array}\right]\right)}^{=n:(A, C)}+\overbrace{\operatorname{rank}\left(\left(\mathrm{s}+\frac{1}{\tau}\right) \mathbb{I}_{\mathrm{m}}\right)}^{\text {detectable }}=n+m .
$$

It should be noticed also that the commandability condition of $(\mathbb{A}, \mathbb{B})$ is preserved thanks to the particular structure of $\mathbb{B}$. However, the observability condition can be lost. The problem of loss of detectability is produced 
when $s=-\frac{1}{\tau}$. Indeed, in this case, we get

$$
\operatorname{rank}(\mathfrak{D e t})<n+m \text { if } p<m
$$

However, if $p \geq m$, it is possible to have $\operatorname{rank}(\mathfrak{D e t})=n+m$; but this depends on the matrices $A, B$ and $C$, but not only on $A$ and $C$ as usually.

To overcome this obstacle, first we can generalize the technique by replacing equation (36) by the following more general one:

$$
\tau \dot{u}+\mathbb{D} u=w,
$$

where $\mathbb{D}$ is an invertible matrix introduced to enhance the observability of $(\mathbb{A}, \mathbb{C})$. Second, we can have some situations where some input variables are accessible (can be measured). That is, we can have as additional output measurement $\bar{y}=C_{u} u$, where $C_{u} \in \mathbb{R}^{\bar{p} \times m}$. In this case, the matrices $\mathbb{A}$ and $\mathbb{C}$ become

$$
\mathbb{A} \triangleq\left[\begin{array}{cc}
-\frac{1}{\tau} \mathbb{D} & 0_{m \times n} \\
B & A
\end{array}\right], \mathbb{C} \triangleq\left[\begin{array}{cc}
0_{p \times m} & C \\
C_{u} & 0_{\bar{p} \times n}
\end{array}\right]
$$

Therefore, we get

$$
\operatorname{rank}(\mathfrak{D e t})=\operatorname{rank}\left(\left[\begin{array}{cc}
s \mathbb{I}_{m}+\frac{1}{\tau} \mathbb{D} & 0 \\
-B & s \mathbb{I}_{n}-A \\
0_{p \times m} & C \\
C_{u} & 0_{\bar{p} \times n}
\end{array}\right]\right) .
$$

Consequently, we have only to choose an invertible matrix $\mathbb{D}$ that compensates the detectability condition on the new $(\mathbb{A}, \mathbb{C})$. Notice that even if we keep $\mathbb{C} \triangleq\left[\begin{array}{ll}0_{p \times m} & C\end{array}\right]$, the matrix $\mathbb{D}$ enable to have the detectability of $(\mathbb{A}, \mathbb{C})$. The new matrix $\mathbb{C}$ is useful if we use the original equation (36) instead of the new more general one (43). Indeed, in such a case, the structure of $\mathbb{C}$ is modified and the number of outputs is now $p+\bar{p}$, for which we can have $p+\bar{p} \geq m$ even in case $p<m$.

\section{EXTENSION TO UNCERTAIN LIPSCHITZ NONLINEAR SYSTEMS}

In order to be more general, an investigation is held on systems in the presence of $\mathcal{L}_{2}$ bounded disturbances. Then, the synthesis will be based on the $\mathcal{H}_{\infty}$ analysis. Hence, consider the system (1) under the effect of additive 
disturbances and Lipschitz nonlinearities described by the following equations:

$$
\begin{gathered}
\dot{x}=(A+\Delta A(t)) x+B u+\phi(x, u)+D \omega \\
y=(C+\Delta C(t)) x+\psi(x, u)+E \omega
\end{gathered}
$$

where $\omega \in \mathbb{R}^{s}$ is the vector of the noises, $D$, and $E$, are $n \times s$, and $p \times s$ real matrices, respectively. The functions $\phi$ and $\psi$ in (44) are globally Lipschitz uniformly with respect to the second variable, that is there exist $\gamma_{\phi}>0$ and $\gamma_{\psi}>0$ such that

$$
\begin{aligned}
& \|\phi(x, u)-\phi(z, u)\| \leq \gamma_{\phi}\|x-z\|, \forall x, z \in \mathbb{R}^{n} \\
& \|\psi(x, u)-\psi(z, u)\| \leq \gamma_{\psi}\|x-z\|, \forall x, z \in \mathbb{R}^{n} .
\end{aligned}
$$

Moreover, without loss of generality let us assume $\phi(0, u)=0$ for all $u \in \mathbb{R}^{m}$. As in the linear case, by applying the transformation $T$ on the system (44), the later takes the following equivalent form:

$$
D:=T D, E:=E, \phi(x, u):=T \phi\left(T^{-1} x, u\right), \psi(x, u):=\psi\left(T^{-1} x, u\right) .
$$

The rest of the parameters are given by (21).

The observer-based controller we consider in this part is under the form :

$$
\begin{gathered}
\dot{\hat{x}}=A \hat{x}+B u+\phi(\hat{x}, u)+L(y-C \hat{x}-\psi(\hat{x}, u)) \\
u=-K \hat{x}
\end{gathered}
$$

where $\hat{x} \in \mathbb{R}^{n}$ is the estimate of $x, K \in \mathbb{R}^{m \times n}$ is the control gain, $L \in \mathbb{R}^{n \times p}$ is the observer gain.

Our problem is then reduced to find simultaneously the observer gain $L$ and the state feedback gain $K$ so that the closed loop system is $\mathcal{H}_{\infty}$ asymptotically stable with attenuation level $\mu>0$. Under the feedback $u=-K \hat{x}$, the closed-loop system has the form:

$$
\left[\begin{array}{l}
\dot{x} \\
\dot{e}
\end{array}\right]=\left[\begin{array}{cc}
(A-B K+\Delta A(t)) & B K \\
(\Delta A(t)-L \Delta C(t)) & (A-L C)
\end{array}\right]\left[\begin{array}{c}
x \\
e
\end{array}\right]+\left[\begin{array}{c}
\phi(x, u) \\
\Delta \phi-L \Delta \psi
\end{array}\right]+\left[\begin{array}{c}
D \\
D-L E
\end{array}\right] \omega
$$

where $e=x-\hat{x}$ represents the estimation error, and

$$
\begin{gathered}
\Delta \phi=\phi(x, u)-\phi(\hat{x}, u) \\
\Delta \psi=\psi(x, u)-\psi(\hat{x}, u) .
\end{gathered}
$$


To deal with the nonlinearities in (47), similarly proceed as in [16]. Hence, according to [27, Lemma 6], the Lipschitz property on $\phi$ and $\psi$ leads to the existence of bounded functions $\phi_{i j}: \mathbb{R}^{n} \times \mathbb{R}^{n} \rightarrow \mathbb{R}, \psi_{i j}$ : $\mathbb{R}^{p} \times \mathbb{R}^{n} \rightarrow \mathbb{R}$ and constants $\underline{\gamma}_{\phi_{i j}}, \bar{\gamma}_{\phi_{i j}}$, for $i, j=1, \ldots, n, \bar{\gamma}_{\psi_{i j}}$ and $\underline{\gamma}_{\psi_{i j}}$, for $i=1, \ldots, p$ and $j=1, \ldots, n$, so that

$$
\underline{\gamma}_{\phi_{i j}} \leq \phi_{i j} \leq \bar{\gamma}_{\phi_{i j}}, \quad \underline{\gamma}_{\psi_{i j}} \leq \psi_{i j} \leq \bar{\gamma}_{\psi_{i j}}
$$

and

$$
\begin{aligned}
\Delta \phi & =\left[\sum_{i=1}^{n} \sum_{j=1}^{n} \phi_{i j}(x, \hat{x}) H_{i j}^{n}\right](x-\hat{x}) \\
\Delta \psi & =\left[\sum_{i=1}^{p} \sum_{j=1}^{n} \psi_{i j}(x, \hat{x}) H_{i j}^{p, n}\right](x-\hat{x}) \\
\phi(x, u) & =\left[\sum_{i=1}^{n} \sum_{j=1}^{n} \phi_{i j}(x, 0) H_{i j}^{n}\right] x
\end{aligned}
$$

where $H_{i j}^{p, n}=e_{p}(i) e_{n}^{T}(j)$. Following the notations in [16], the system (47) can be rewritten under the form

$$
\left[\begin{array}{l}
\dot{x} \\
\dot{e}
\end{array}\right]=\left[\begin{array}{cc}
(\mathcal{A}(\Lambda)-B K+\Delta A(t)) & B K \\
(\Delta A-L \Delta C(t)) & (\mathcal{A}(\Upsilon)-L \mathcal{C}(\Xi))
\end{array}\right]\left[\begin{array}{l}
x \\
e
\end{array}\right]+\left[\begin{array}{c}
D \\
D-L E
\end{array}\right] \omega
$$

which takes the compact form

$$
\dot{z}=\Pi_{1}(\Lambda, \Upsilon, \Xi) z+\Pi_{2} \omega
$$

where $z^{T}=\left[x^{T} e^{T}\right]$ and the detailed expression of the matrices in $\Pi_{1}(\Lambda, \Upsilon, \Xi)$ derived from the nonlinerities are respectively

$$
\begin{gathered}
\mathcal{A}(\Lambda):=A+\Lambda=A+\sum_{i=1}^{n} \sum_{j=1}^{n} \phi_{i j}(x, 0) H_{i j}^{n} \\
\mathcal{A}(\Upsilon):=A+\Upsilon=A+\sum_{i=1}^{n} \sum_{j=1}^{n} \phi_{i j}(x, \hat{x}) H_{i j}^{n} \\
\mathcal{C}(\Xi):=C+\Xi=C+\sum_{i=1}^{p} \sum_{j=1}^{n} \psi_{i j}(x, \hat{x}) H_{i j}^{p, n} .
\end{gathered}
$$

and the parameters $\Lambda$ and $\Upsilon$ (resp. $\Xi$ ) belong to bounded convex set $\mathcal{H}_{n}$ (resp. $\mathcal{H}_{p}$ ) for which the set of vertices is defined by:

$$
\mathcal{V}_{\mathcal{H} n}=\left\{\Pi \in \mathbb{R}^{n \times n}, \Pi_{i j} \in\left\{\underline{\gamma}_{\phi_{i j}}, \bar{\gamma}_{\phi_{i j}}\right\}\right\},\left(\text { resp. } \mathcal{V}_{\mathcal{H} p}=\left\{\Gamma \in \mathbb{R}^{p \times n}, \Gamma_{i j} \in\left\{\underline{\gamma}_{\psi_{i j}}, \bar{\gamma}_{\psi_{i j}}\right\}\right\}\right)
$$

Our objective consists in finding matrices $K$ and $L$ so that the augmented closed-loop system (51) with $\omega(t)=0$ is asymptotically stable; and the effect of $\omega(t)$ on the tracking error $Z(t)=H x(t)$, where $H$ is a known matrix 
of appropriate dimension, is attenuated in the $\mathcal{H}_{\infty}$ sense. More precisely, it is required that

$$
\|Z\|_{2}<\mu\|\omega\|_{2}, \forall \omega \in \mathcal{L}_{2}[0, \infty)
$$

where $\mu>0$ is the disturbance attenuation level to be determined. To this end, let's consider the following index $\mathcal{J}$

$$
\mathcal{J}=\int_{0}^{\infty}\left[Z(t)^{T} Z(t)-\mu^{2} \omega(t)^{T} \omega(t)\right] d t
$$

Under zeros-initial conditions, the Lyapunov function satisfies $V(0)=0$ and $V(\infty) \geq 0$, which leads to

$$
\begin{array}{r}
\mathcal{J}=\int_{0}^{\infty}\left[Z^{T}(t) Z(t)-\mu^{2} \omega(t)^{T} \omega(t)+\dot{V}(t)\right] d t-V(\infty) \\
\leq \int_{0}^{\infty}\left[Z^{T}(t) Z(t)-\mu^{2} \omega(t)^{T} \omega(t)+\dot{V}(t)\right] d t
\end{array}
$$

To satisfy the attenuation level in (53), it suffices that inequality (56) holds :

$$
Z^{T}(t) Z(t)-\mu^{2} \omega(t)^{T} \omega(t)+\dot{V}(t)<0, \forall t \in[0, \infty)
$$

Derived from the same Lyapunov function candidate as previously, that is

$$
V(z)=z^{T} \operatorname{diag}\{P, R\} z
$$

Therefore,

$$
\dot{V}=\operatorname{He}\left(z^{T} \Pi_{1}^{T}(\Lambda, \Upsilon, \Xi) \operatorname{diag}\{P, R\} z+\omega^{T} \Pi_{2}^{T} \operatorname{diag}\{P, R\} z\right)
$$

According to (2) and using Young inequality, $\dot{V}$ can be upper bounded as follows:

$$
\dot{V} \leq\left[\begin{array}{c}
x \\
e \\
\omega
\end{array}\right]^{T}\left[\begin{array}{ccc}
\Omega_{11} & P B K & P D \\
(\star) & \Omega_{22} & R(D-L E) \\
(\star) & (\star) & 0
\end{array}\right]\left[\begin{array}{l}
x \\
e \\
\omega
\end{array}\right]
$$

where

$$
\begin{aligned}
\Omega_{11}= & {\left[(\mathcal{A}(\Lambda)-B K)^{T} P+P(\mathcal{A}(\Lambda)-B K)\right] } \\
& +\left[\left(\epsilon_{1}+\epsilon_{2}\right) N_{1}^{T} N_{1}+\epsilon_{3} N_{2}^{T} N_{2}+\frac{1}{\epsilon_{1}} P M_{1} M_{1}^{T} P\right] \\
\Omega_{22}= & {\left[(\mathcal{A}(\Upsilon)-L \mathcal{C}(\Xi))^{T} R+R(\mathcal{A}(\Upsilon)-L \mathcal{C}(\Xi))\right] } \\
& +\left[\frac{1}{\epsilon_{2}} R M_{1} M_{1}^{T} R+\frac{1}{\epsilon_{3}} R L M_{2} M_{2}^{T} L^{T} R\right]
\end{aligned}
$$


and $\epsilon_{1}, \epsilon_{2}, \epsilon_{3}$ are positive real constants. Inequality (56) is then satisfied if the following quadratic form

$$
\left[\begin{array}{l}
x \\
e \\
\omega
\end{array}\right]^{T}\left[\begin{array}{ccc}
\Omega_{11}+H^{T} H & P B K & P D \\
(\star) & \Omega_{22} & R(D-L E) \\
(\star) & (\star) & -\mu^{2} I
\end{array}\right]\left[\begin{array}{c}
x \\
e \\
\omega
\end{array}\right]<0
$$

holds for all $(\Lambda, \Upsilon, \Xi) \in \mathcal{H}_{n} \times \mathcal{H}_{n} \times \mathcal{H}_{p}$, or equivalently

$$
\Omega(\Lambda, \Upsilon, \Xi)<0, \forall(\Lambda, \Upsilon, \Xi) \in \mathcal{H}_{n} \times \mathcal{H}_{n} \times \mathcal{H}_{p}
$$

with

$$
\Omega(\Lambda, \Upsilon, \Xi)=\left[\begin{array}{cccccc}
\tilde{\Omega}_{11} & P B K & P M_{1} & 0 & 0 & P D \\
(\star) & \tilde{\Omega}_{22} & 0 & R M_{1} & \hat{L} M_{2} & R D-\hat{L} E \\
(\star) & (\star) & -\epsilon_{1} I & 0 & 0 & 0 \\
(\star) & (\star) & (\star) & -\epsilon_{2} I & 0 & 0 \\
(\star) & (\star) & (\star) & (\star) & -\epsilon_{3} I & 0 \\
(\star) & (\star) & (\star) & (\star) & (\star) & -\delta I
\end{array}\right],
$$

and

$$
\begin{aligned}
& \tilde{\Omega}_{11}=\operatorname{He}\{P \mathcal{A}(\Lambda)-P B K\}+H^{T} H+\left(\epsilon_{1}+\epsilon_{2}\right) N_{1}^{T} N_{1}+\epsilon_{3} N_{2}^{T} N_{2} \\
& \tilde{\Omega}_{22}=\operatorname{He}\{R \mathcal{A}(\Upsilon)-\hat{L} \mathcal{C}(\Xi)\}, \hat{L}=R L, \delta=\mu^{2} .
\end{aligned}
$$

\subsection{Linearization of (61) via equality constraint method (ECM)}

As a start begins by presenting sufficient conditions including an equality constraint to ensure the stability of the system (47) and the $\mathcal{H}_{\infty}$ criterion (53). In order to linearize (61), the approach in [18] is used.

\section{Theorem 4.1}

System (44) is asymptotically stabilizable by (46) if there exist positive definite matrices $P, R \in \mathbb{R}^{n \times n}$, matrices $\hat{K} \in \mathbb{R}^{m \times n}, \hat{L} \in \mathbb{R}^{n \times p}$ and $\hat{P} \in \mathbb{R}^{m \times m}$ so that the following LMI optimization problem

$\operatorname{minimize}(\delta)$

subject to the following set of LMIs and equality constraint 


$$
\left[\begin{array}{cccccc}
\mathcal{L}_{11}(\Lambda) & B \hat{K} & P M_{1} & 0 & 0 & P D \\
(\star) & \mathcal{L}_{22}(\Upsilon, \Xi) & 0 & R M_{1} & \hat{L} M_{2} & R D-\hat{L} E \\
(\star) & (\star) & -\epsilon_{1} I & 0 & 0 & 0 \\
(\star) & (\star) & (\star) & -\epsilon_{2} I & 0 & 0 \\
(\star) & (\star) & (\star) & (\star) & -\epsilon_{3} I & 0 \\
(\star) & (\star) & (\star) & (\star) & (\star) & -\delta I
\end{array}\right]<0
$$

for all $(\Lambda, \Upsilon, \Xi) \in \mathcal{H}_{n} \times \mathcal{H}_{n} \times \mathcal{H}_{p}$,

$P B=B \hat{P}$

where

$$
\begin{aligned}
\mathcal{L}_{11}(\Lambda) & =\operatorname{He}\{P \mathcal{A}(\Lambda)-B \hat{K}\}+H^{T} H+\left(\epsilon_{1}+\epsilon_{2}\right) N_{1}^{T} N_{1}+\epsilon_{3} N_{2}^{T} N_{2} \\
\mathcal{L}_{22}(\Upsilon, \Xi) & =\operatorname{He}(R \mathcal{A}(\Upsilon)-\hat{L} \mathcal{C}(\Xi))
\end{aligned}
$$

is feasible. The stabilizing observer-based control gains are given by $K=\hat{P}^{-1} \hat{K}$ and $L=R^{-1} \hat{L}$ and the optimal disturbance attenuation level is given by $\mu_{\min }=\sqrt{\delta}$.

\subsection{Linearization of (61) via Young's relation based approach}

This section is devoted to the presentation of the Young's relation based approach, for nonlinear systems, to obtain a set of bilinear conditions for the robust observer-based controller design problem, which become linear matrix inequalities by freezing some scalars. Note that this method does not depend on $m$ and works successfully for all $m \leq n$, and without imposing the particular stucture of $B$. As in the linear case [15, 14], this method is based on a judicious use of the Young's relation to linearize the classical BMI problem (61).

\section{Theorem 4.2}

System (44) is asymptotically stabilizable by (46) if for fixed scalars $\epsilon_{2}>0, \epsilon_{3}>0$ and $\epsilon_{4}>0$, there exist two positive definite matrices $\mathcal{Z} \in \mathbb{R}^{n \times n}, R \in \mathbb{R}^{n \times n}$, two matrices $\hat{K} \in \mathbb{R}^{m \times n}, \hat{L} \in \mathbb{R}^{n \times p}$, and a scalar $\epsilon_{1}>0$ so that the following LMI optimization problem has a solution

$\operatorname{minimize}(\delta)$

subject to the following set of LMIs 


$$
\left[\begin{array}{cc}
\mathcal{K}_{11}(\Lambda, \Upsilon, \Xi) & \mathcal{K}_{12} \\
(\star) & -\operatorname{diag}\left\{\frac{1}{\epsilon_{4}} \mathcal{Z}, \epsilon_{4} \mathcal{Z}, \epsilon_{1} I, \epsilon_{2} I, \frac{1}{\epsilon_{2}}, \epsilon_{3} I, \frac{1}{\epsilon_{3}} I, \delta I\right\}
\end{array}\right]<0
$$

$$
\text { for all }(\Lambda, \Upsilon, \Xi) \in \mathcal{H}_{n} \times \mathcal{H}_{n} \times \mathcal{H}_{p}
$$

with

$$
\begin{gathered}
\mathcal{K}_{12}=\left[\begin{array}{cccccccc}
B \hat{K} & 0 & \mathcal{Z} N_{1}^{T} & 0 & \mathcal{Z} N_{1}^{T} & 0 & \mathcal{Z} N_{2}^{T} & D \\
0 & 0 & 0 & 0 & 0 & 0 & 0 & 0 \\
0 & I & 0 & R M_{1} & 0 & \hat{L} M_{2} & 0 & R D-\hat{L} E
\end{array}\right] \\
\mathcal{K}_{11}(\Lambda, \Upsilon, \Xi)=\left[\begin{array}{ccc}
\mathcal{K}_{11}^{11}(\Lambda) & \mathcal{Z} H^{T} & 0 \\
(\star) & -I & 0 \\
(\star) & (\star) & \mathcal{K}_{11}^{33}(\Upsilon, \Xi)
\end{array}\right] \\
\mathcal{K}_{11}^{11}(\Lambda)=\operatorname{He}(\mathcal{A}(\Lambda) \mathcal{Z}-B \hat{K})+\epsilon_{1} M_{1} M_{1}^{T} \\
\mathcal{K}_{11}^{33}(\Upsilon, \Xi)=\operatorname{He}(R \mathcal{A}(\Upsilon)-\hat{L} \mathcal{C}(\Xi)) .
\end{gathered}
$$

Hence, the $\mathcal{H}_{\infty}$ stabilizing observer-based control gains are given by $K=\hat{K} \mathcal{Z}^{-1}$ and $L=R^{-1} \hat{L}$, and the optimal disturbance attenuation level is given by $\mu_{\min }=\sqrt{\delta}$.

\subsection{Linearization of (61) via the new two-steps algorithm (NTSA)}

Starting from inequality (10). Taking into account the detailed structure of the term $P B K$, namely (24), and the structure (26), inequalities (61) become after using the change of variables $\hat{K}=P_{11} K, \hat{L}=R L, \delta=\mu^{2}$ :

$$
\left[\begin{array}{cccccc}
\tilde{\Omega}_{11} & {\left[\begin{array}{c}
\hat{K} \\
S^{T} \hat{K}
\end{array}\right]} & P M_{1} & 0 & 0 & P D \\
(\star) & \tilde{\Omega}_{22} & 0 & R M_{1} & \hat{L} M_{2} & R D-\hat{L} E \\
(\star) & (\star) & -\epsilon_{1} I & 0 & 0 & 0 \\
(\star) & (\star) & (\star) & -\epsilon_{2} I & 0 & 0 \\
(\star) & (\star) & (\star) & (\star) & -\epsilon_{3} I & 0 \\
(\star) & (\star) & (\star) & (\star) & (\star) & -\delta I
\end{array}\right]<0
$$


for all $(\Lambda, \Upsilon, \Xi) \in \mathcal{V}_{\mathcal{H} n} \times \mathcal{V}_{\mathcal{H} n} \times \mathcal{V}_{\mathcal{H} p}$, and

$$
\tilde{\Omega}_{11}=\operatorname{He}\left\{P \mathcal{A}(\Lambda)-\left[\begin{array}{c}
\hat{K} \\
S^{T} \hat{K}
\end{array}\right]\right\}+H^{T} H+\left(\epsilon_{1}+\epsilon_{2}\right) N_{1}^{T} N_{1}+\epsilon_{3} N_{2}^{T} N_{2} .
$$

At this stage, we proceed as in Subsection 3.4. We consider the stabilization problem of (44) by a static state feedback $u=-\mathcal{K} x$. System (44a) can be written in the closed-loop form (by taking into account the rewrite of $\phi$ in (50)):

$$
\dot{x}=\left(\mathcal{A}(\Lambda)-B \mathcal{K}+M_{1} F_{1}(t) N_{1}\right) x+D \omega
$$

System (70) is is globally $\mathcal{H}_{\infty}$ asymptotically stable with a minimum attenuation level $\nu$ by the Lyapunov function $V(x)=x^{T} \mathcal{P} x$ if the following inequalities hold:

$$
\begin{gathered}
(\mathcal{A}(\Lambda)-B \mathcal{K})^{T} \mathcal{P}+\mathcal{P}(\mathcal{A}(\Lambda)-B \mathcal{K}) \\
+N_{1}^{T} F_{1}^{T}(t) M_{1}^{T} \mathcal{P}+\mathcal{P} M_{1} F_{1}(t) N_{1}+H^{T} H+\nu^{-2} \mathcal{P} D D^{T} \mathcal{P}<0, \forall \Lambda \in \mathcal{V}_{\mathcal{H} n}
\end{gathered}
$$

Inequalities (71) can be easily linearized as in (31). We deduce that (70) is globally $\mathcal{H}_{\infty}$ asymptotically stable if the following LMI conditions are fulfilled:

$$
\left[\begin{array}{cccc}
\operatorname{He}(\mathcal{A}(\Lambda) \mathcal{Z}-B \overline{\mathcal{K}})+\epsilon M_{1} M_{1}^{T} & \mathcal{Z} N_{1}^{T} & \mathcal{Z} H^{T} & D \\
(\star) & -\epsilon I & 0 & 0 \\
(\star) & (\star) & -I & 0 \\
(\star) & (\star) & (\star) & -\hat{\delta} I
\end{array}\right]<0, \forall \Lambda \in \mathcal{V}_{\mathcal{H} n}
$$

where $\overline{\mathcal{K}}=\mathcal{K} \mathcal{Z}, \hat{\delta}=\nu^{2}$ and $\mathcal{Z}^{-1}=\mathcal{P}=\left[\begin{array}{ll}\mathcal{P}_{11} & \mathcal{P}_{12} \\ \mathcal{P}_{12}^{T} & \mathcal{P}_{22}\end{array}\right]$. The rest of our approach is reported in the following new two-steps algorithm:

\section{Algorithm 5: general algorithm for nonlinear systems}

step 1: Solve the optimization problem: minimize $\delta$ subject to LMI (68) with the decision variables $\mathcal{Z}, R, \hat{K}, \hat{L}$, and $\epsilon_{1}>0$ and go to step 2;

step 2: If LMI (68) is found feasible, then compute the observer-based controller gains as $K=\hat{K}^{T} \mathcal{Z}^{-1}$,

$$
L=R^{-1} \hat{L}^{T} \text { and } \mu_{\min }=\sqrt{\delta} \text {. Otherwise, go to step } 3 ;
$$


step 3: Solve the optimization problem: minimize $\hat{\delta}$ subject to LMI (72) with the decision variables $\mathcal{Z}, \overline{\mathcal{K}}$, and $\epsilon>0$, and go to step 4 ;

step 4: Compute $\bar{S}$ by $\bar{S}=\mathcal{P}_{11}^{-1} \mathcal{P}_{12}$, put $S=\Theta \bar{S}$, with $\Theta=\operatorname{diag}\left(\alpha_{1}, \alpha_{2}, \ldots, \alpha_{m}\right)$ and go to step 5;

step 5: Solve the optimization problem: minimize $\delta$ subject to (69) with $S=\Theta \bar{S}$ by using the gridding method on $\alpha_{i}, i=1, \ldots, m$, with the decision variables $P_{11}, P_{22}, R, \hat{K}, \hat{L}$ and $\epsilon_{i}, i=1,2,3$, and go to step 6 ;

step 6 Compute the observer-based controller gains as $K=P_{11}^{-1} \hat{K}, L=R^{-1} \hat{L}$ and the optimal disturbance attenuation level as $\mu_{\min }=\sqrt{\delta}$.

\section{NUMERICAL EXAMPLES AND COMPARISONS}

In this section, numerical examples are presented to show the validity and effectiveness of the proposed design methodology.

\subsection{Example 1}

Consider the same example as that in [18]. The system has the following parameters:

$$
\begin{gathered}
A=\left[\begin{array}{ccc}
1 & 1 & 1 \\
0 & -2 & 1 \\
1 & -2 & -5
\end{array}\right], B=\left[\begin{array}{ll}
1 & 0 \\
0 & 1 \\
0 & 0
\end{array}\right] \\
C=\left[\begin{array}{lll}
1 & 0 & 1
\end{array}\right], D=0 \\
\Delta A(t)=\left[\begin{array}{ccc}
0 & 0 & a(t) \\
0 & b(t) & 0 \\
c(t) & 0 & 0
\end{array}\right], \Delta C(t)=\left[\begin{array}{lll}
0 & d(t) & 0
\end{array}\right]
\end{gathered}
$$

where $a(t) \leq \alpha, b(t) \leq \beta, c(t) \leq \gamma$ and $d(t) \leq \delta$. The uncertainties can be rewritten under the form (2) with

$$
\begin{gathered}
M_{1}=M_{2}=I \\
F_{1}(t)=\left[\begin{array}{ccc}
\frac{a(t)}{\alpha} & 0 & 0 \\
0 & \frac{b(t)}{\beta} & 0 \\
0 & 0 & \frac{c(t)}{\gamma}
\end{array}\right]
\end{gathered}
$$




$$
F_{2}(t)=\frac{d(t)}{\delta}, \quad N_{1}=\left[\begin{array}{lll}
0 & 0 & \alpha \\
0 & \beta & 0 \\
\gamma & 0 & 0
\end{array}\right], \quad N_{2}=\left[\begin{array}{lll}
0 & \delta & 0
\end{array}\right]
$$

5.1.1. Case 1 Assume that $\alpha=\beta=\gamma=\delta$. Testing the feasibility of LMIs (11), (12)-(13), (14) and the new design algorithm. To do that, we search for the maximum value of $\alpha, \alpha_{\max }$, tolerated by each method. The superiority of the new design Algorithm 3, is quite clear from Table I.

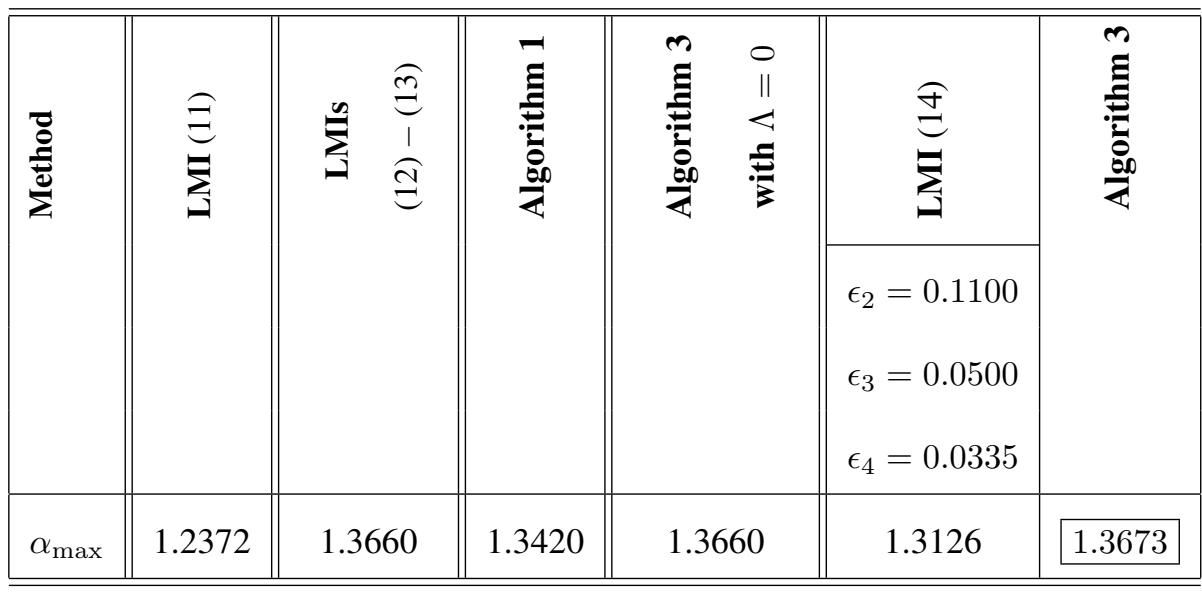

Table I. Case 1: superiority of the new LMI design procedure

5.1.2. Case 2: modification of $A$ and $B$ It is clear that all the above mentioned methods depend strongly on the input matrix $B$. For this, $B$ is modified and the feasibility of each method is tested in order to look for the tolerated maximum value of $\alpha_{\max }$. It is assumed that there is only one input to stabilize the system. We also modify the last diagonal element of $A$. Indeed, we take the following matrices:

$$
A=\left[\begin{array}{ccc}
1 & 1 & 1 \\
0 & -2 & 1 \\
1 & -2 & 0
\end{array}\right], B=\left[\begin{array}{l}
1 \\
0 \\
0
\end{array}\right]
$$

Under these new considerations, new values of $\alpha_{\max }$ are obtained for each method. The new values are illustrated in Table II. 


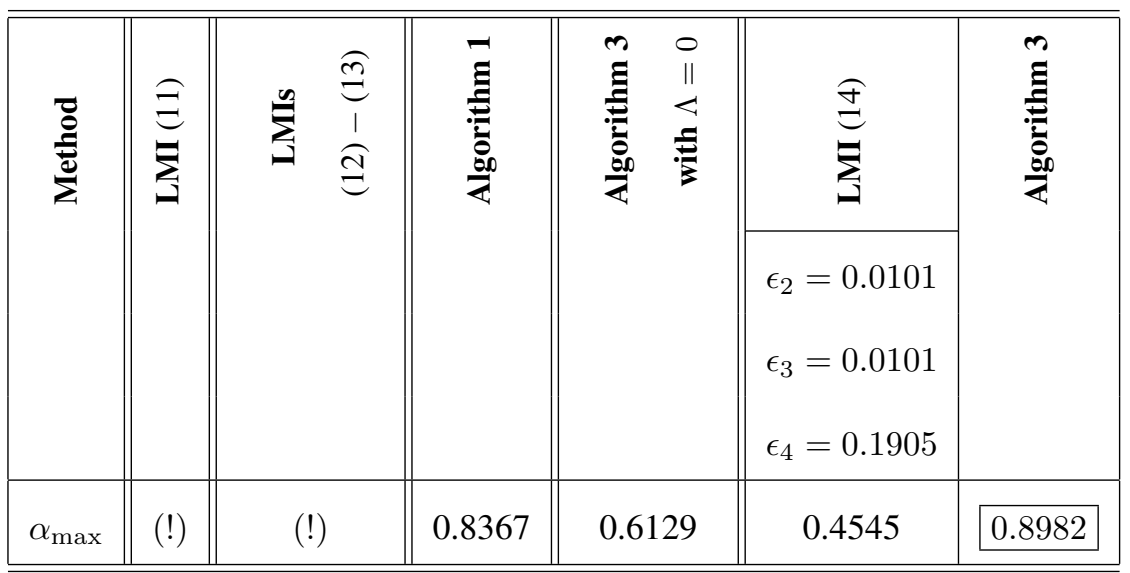

Table II. Case 2: superiority of the new LMI design procedure

Notice that the symbol (!) means that LMIs (11) and (12)-(13) are unfeasible even without uncertainties. Indeed, as mentioned in (35), the diagonal block $A_{22}^{2}=0$ is not Schur stable. Hence, the LMIs (11) and (12)(13) cannot be applied.

\subsection{Example 2 (DC motor)}

This example is introduced in order to show the effectiveness of the proposed design method in the case of systems with uncertainty in the $B$ matrix. For this, we consider the DC motor model given in [12] and described by the following matrices:

$$
A=\left[\begin{array}{cc}
-\frac{b}{J} & \frac{K_{m}}{J} \\
-\frac{K_{m}}{L_{m}} & -\frac{R_{m}}{L_{m}}
\end{array}\right], B=\left[\begin{array}{c}
0 \\
\frac{1}{L_{m}}
\end{array}\right], C=\left[\begin{array}{ll}
1 & 0
\end{array}\right] .
$$

The parameters of the DC motor are presented in Table III.

The electric inductance $L_{m}$ is assumed to be time varying and uncertain, which leads to an uncertain system. Since the equations depend only on $\frac{1}{L_{m}}$, then it is assumed that $\frac{1}{L_{m}}$ is affected by an additive uncertainty as follows:

$$
\frac{1}{L_{m}(t)}=\frac{1}{L_{m}^{0}}+\Delta(t)
$$

where $\Delta(t)$ satisfies the inequality

$$
|\Delta(t)| \leq \Delta_{\max }
$$




\begin{tabular}{llll}
\hline Moment of inertia of the rotor & $J=0.01 \quad \mathrm{~kg} . \mathrm{m}^{2}$ \\
Motor viscous friction constant & $b=0.1 \quad \mathrm{~N} . \mathrm{m} . \mathrm{s}$ \\
Electromotive force constant & $K_{e}=K_{m}=0.01 \quad \mathrm{~V} / \mathrm{rad} / \mathrm{s}$ \\
Motor torque constant & $K_{t}=K_{m}=0.01 \quad \mathrm{~N} . \mathrm{m} / \mathrm{Amp}$ \\
Electric resistance & $R_{m}=1 \quad \Omega$ \\
Electric inductance & $L_{m}^{0}=0.5 \quad H$ \\
\hline
\end{tabular}

Table III. The DC motor model parameters

and $L_{m}^{0}=0.5 \mathrm{H}$ is the nominal value of the electric inductance.

Now, we search for the largest value $\Delta_{\max }\left(\right.$ then $\delta_{\max }$ ) tolerated by all the previous methods. The results are summarized in Table IV.

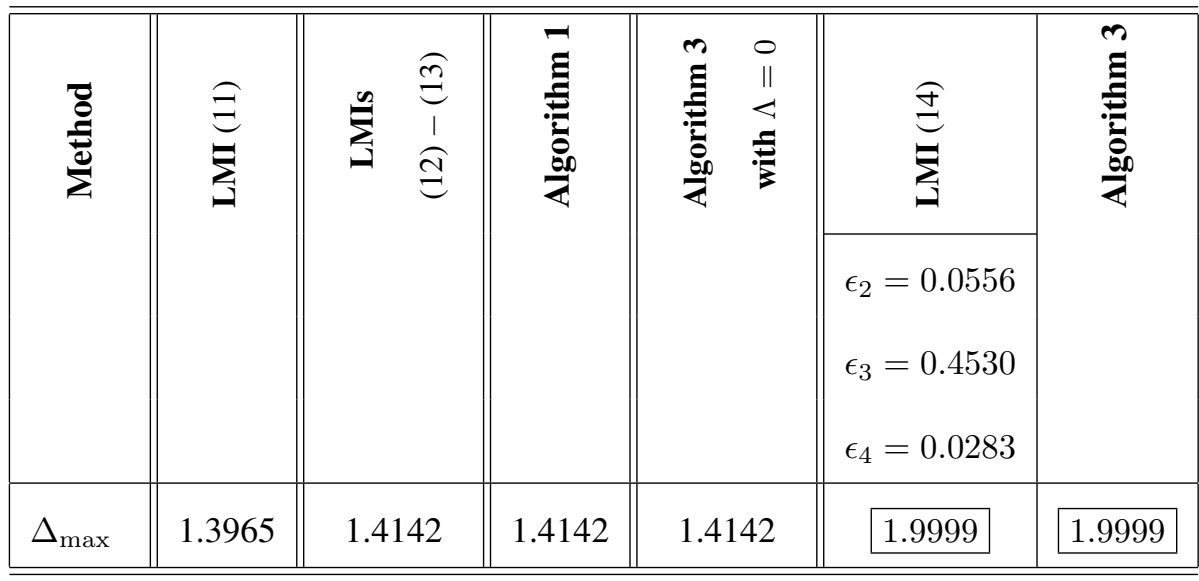

Table IV. Example 2: superiority of the Algorithm 4

\subsection{Monte-Carlo evaluation in uncertainty-free case}

The aim consists in evaluating numerically the necessary conditions required by each method. For this, we generate randomly 1000 stabilizable and detectable linear systems of dimension $n=3, p=1$ and $m$ ranging from 1 to $n$. After transformation of the system to have a matrix $B$ under the form (22), we obtain the results 
of Table III, which gives the percentage of systems for which the different methods addressed in this paper succeeded for each value of $m$.

\begin{tabular}{|c|c|c|c|c|c|c|}
\hline 胥 & $\underset{\Xi}{\Xi}$ & 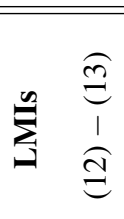 & 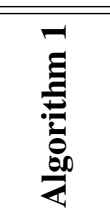 & 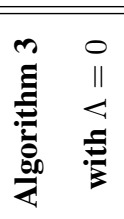 & $\frac{\stackrel{\Im}{\Xi}}{\sum_{\Xi}^{\Xi}}$ & 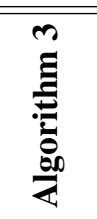 \\
\hline$m=1$ & $15.8 \%$ & $47.8 \%$ & $56.9 \%$ & $48.3 \%$ & $100 \%$ & $100 \%$ \\
\hline$m=2$ & $50.1 \%$ & $69.0 \%$ & $70.6 \%$ & $72.6 \%$ & $100 \%$ & $100 \%$ \\
\hline$m=3$ & $100 \%$ & $100 \%$ & $100 \%$ & $100 \%$ & $100 \%$ & $100 \%$ \\
\hline
\end{tabular}

Table V. Superiority of the new LMI design procedure in the uncertainty-free case

Notice that even if the system is treated without transformation of $B$ under the form (22), the Young's relation based approach (LMI (14)) remains better. The results of this case are provided in Table V.

It is quite clear from Tables V and VI that the proposed new Algorithm 4, combining LMI (14) and Algorithm 3, is the least conservative and succeeds for all $m \leq n$, while the results obtained by the other methods depend on the value of $m$.

\begin{tabular}{|l||c||c||c||c||}
\hline \hline Method & LMI (11) & LMIs (12)-(13) & Algorithm 1 & LMI (14) \\
\hline$m=1$ & $13.8 \%$ & $57.5 \%$ & $54.3 \%$ & $100 \%$ \\
\hline$m=2$ & $50.8 \%$ & $80.5 \%$ & $68.2 \%$ & $100 \%$ \\
\hline$m=3$ & $100 \%$ & $100 \%$ & $100 \%$ & $100 \%$ \\
\hline \hline
\end{tabular}

Table VI. Superiority of the new LMI design procedure in the uncertainty-free case

\subsection{Example 3: Validation of Algorithm 5}

In order to validate the approaches proposed in Section 4, all the optimization problems (65), (68)-(66), Algorithm 5, and the classical two-steps method (TSM) are tested on the system described by the following 
parameters taken from [9]:

$$
\begin{gathered}
A=\left[\begin{array}{ccc}
\frac{1}{3} & \frac{3}{2} & 0 \\
\frac{1}{2} & \frac{3}{5} & 1 \\
1 & -1 & \frac{1}{3}
\end{array}\right], B=\left[\begin{array}{cc}
0 & \frac{1}{2} \\
\frac{1}{3} & 0 \\
0 & 0
\end{array}\right], C=\left[\begin{array}{ccc}
-\frac{1}{9} & \frac{1}{2} & 0 \\
\frac{1}{5} & \frac{1}{6} & \frac{1}{9}
\end{array}\right], H=C, \\
\phi(x)=\left[\begin{array}{lll}
0 & \frac{1}{7} \frac{x_{1}}{1+x_{1}^{2}} & 0
\end{array}\right]^{T}, D=0.1 I_{3} .
\end{gathered}
$$

By applying the transformation $T=\left[\begin{array}{lll}0 & 3 & 0 \\ 2 & 0 & 0 \\ 0 & 0 & 1\end{array}\right]$ on the previous system, the following new parameters $A, B$, $C$ and $\phi($.$) are reached:$

$$
\begin{gathered}
A:=\left[\begin{array}{ccc}
\frac{3}{5} & \frac{3}{4} & 3 \\
1 & \frac{1}{3} & 0 \\
-\frac{1}{3} & \frac{1}{2} & \frac{1}{3}
\end{array}\right], B:=\left[\begin{array}{cc}
1 & 0 \\
0 & 1 \\
0 & 0
\end{array}\right], C:=\left[\begin{array}{ccc}
\frac{1}{6} & -\frac{1}{18} & 0 \\
\frac{1}{18} & \frac{1}{10} & \frac{1}{9}
\end{array}\right], H=C, \\
\phi(x):=\left[\begin{array}{lll}
\frac{6}{7} \frac{x_{2}}{4+x_{2}^{2}} & 0 & 0
\end{array}\right]^{T}, D=0.1 T .
\end{gathered}
$$

According to [27, Lemma 6], the set of vertices $\mathcal{V}_{\mathcal{H}}$ is defined as:

$$
\mathcal{V}_{\mathcal{H} n}=\left\{\left[\begin{array}{ccc}
0 & \frac{-3}{112} & 0 \\
0 & 0 & 0 \\
0 & 0 & 0
\end{array}\right],\left[\begin{array}{ccc}
0 & \frac{3}{14} & 0 \\
0 & 0 & 0 \\
0 & 0 & 0
\end{array}\right]\right\}
$$

With these parameters, except the approach based on the classical two-steps method (TSM), which unfortunately does not provide solutions, all the approaches presented in Section 4 perform successfully. LMI (65) under constraint equality (66) of Theorem 4.1 provides solutions for optimal value $\mu_{\min }(\mathrm{ECM})=$ 10, 9050. Using the gridding method, for $\epsilon_{4}=0.0003$, LMI (68) was found feasible. The corresponding optimal disturbance attenuation level is given by $\mu_{\min }$ (Young) $=4.71 \times 10^{-4}$. As compared to (NTSA) Algorithm 5, for $\alpha_{1}=\alpha_{2}=1$, the optimal value of the disturbance attenuation level is $\mu_{\min }^{*}(\mathrm{NTSA})=4.33 \times 10^{-5}$. The simulation results when applying the gridding method (with a length of discretization equal to $h=0.001$ ) show that the first instant making Algorithm 5 feasible when $\kappa$ browsing $]-1,+1[$ corresponds to $\kappa=0.593$. These results show also that Algorithm 5 performs successfully for all $\kappa \in\left[0.593,1\left[\right.\right.$. The evolution of $\mu_{\min }(\mathrm{NTSA})$ 
with respect to $\kappa \in\left[0.593,1\right.$ [ is illustrated in Figure 1 . The optimal value $\mu_{\min }^{*}$ (NTSA) is obtained by

$$
\mu_{\min }^{*}(\mathrm{NTSA})=\min _{\kappa \in[0.593,1[} \mu_{\min }(\mathrm{NTSA})(\kappa)
$$

The later is attained for $\kappa=0.6180$ (or equivalently, for $\alpha=1$ ). It is clear that Algorithm 5 performs better than

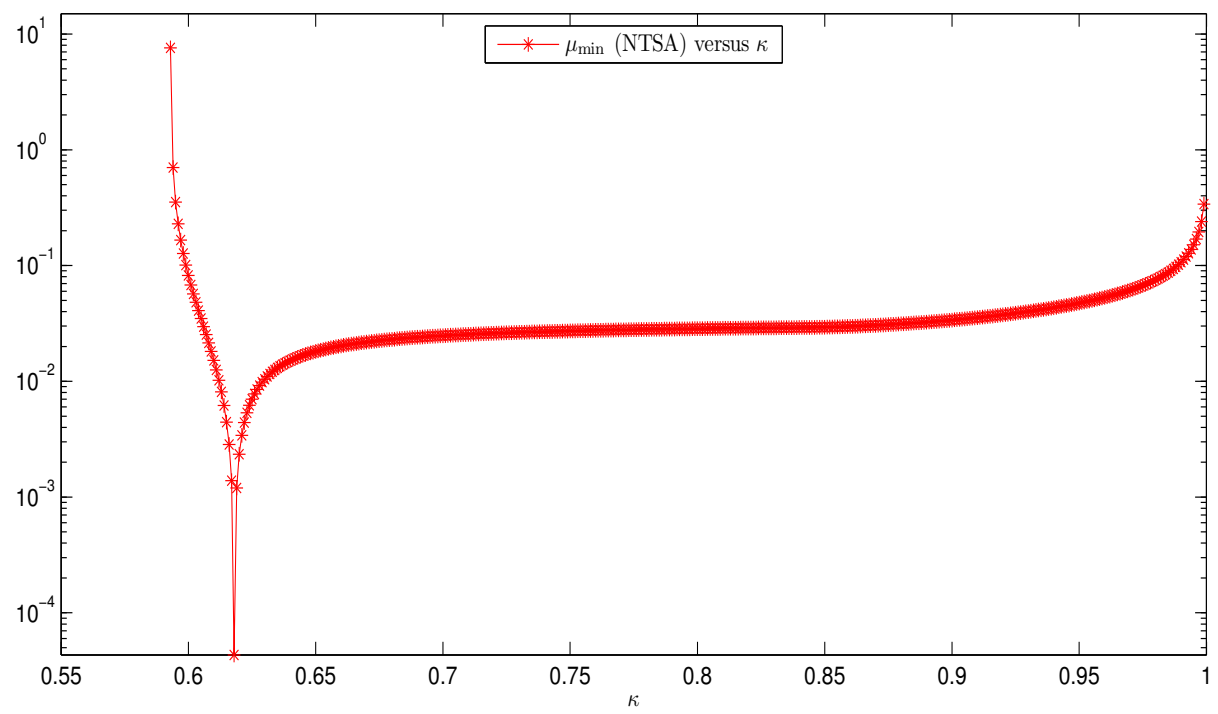

Figure 1. Evolution of $\mu_{\min }$ (NTSA) via the gridding method

the other LMI design methods.

\subsection{Evaluation with respect to the $\mathcal{H}_{\infty}$ attenuation level}

To evaluate the conservatism of the proposed (NTSM) Algorithm 5 as compared to Young based approach, equality constraint based method (ECM), and the classical two-steps method (TSM) we take again the linear systems in Example 1, with $\Delta A(t)=\Delta C(t)=0$, under the effect of additive noises, represented by random matrices $D, E$ and $H$. The aim here consists in evaluating the better index performance tolerated by each method. We randomly gererate 1000 systems via a Monte Carlo simulation and compute the percentage of feasible optimization problem for each method. The three approaches: Young based approach (LMIs (68)), equality constraint based method (ECM) (represented by LMI (65)-(66)), and the new two-steps method (NTSM) (or Algorithm 5), provide solutions for $100 \%$ of systems, while the classical two-steps method performs successfully in $76 \%$ cases. In order to boost the comparison, we assume that the values of the objective function are fixed to be all lower than $\mu_{\max }=10$. The results a re s ummarized in Table V II. A s s hown in 


\begin{tabular}{|l||c||c||c||c||}
\hline \hline Method & ECM & Young & Algo. 5 & TSM \\
\hline \hline Feasible LMIs & $100 \%$ & $100 \%$ & $100 \%$ & $76 \%$ \\
\hline \hline$\mu_{\max }<10$ & $37.1 \%$ & $100 \%$ & $100 \%$ & $37.4 \%$ \\
\hline \hline$\mu_{\min }\left(\right.$ Algo. 5) $<\mu_{\min }($ Method) & $100 \%$ & $94 \%$ & - & $100 \%$ \\
\hline \hline$\mu_{\min }($ Young $)<\mu_{\min }($ Method) & $100 \%$ & - & $6 \%$ & $94.6 \%$ \\
\hline \hline$\mu_{\min }(\mathrm{ECM})<\mu_{\min }($ Method) & - & $0 \%$ & $0 \%$ & $10 \%$ \\
\hline \hline$\mu_{\min }(\mathrm{TSM})<\mu_{\min }($ Method) & $90 \%$ & $5.4 \%$ & $0 \%$ & - \\
\hline \hline
\end{tabular}

Table VII. Superiority of the proposed LMI methodology in $\mathcal{H}_{\infty}$ case

Table VII, Algorithm 5 and the Young based approach provide solution for the largest percentage of systems. Note that among the LMIs (65) under (66) found feasible, $100 \%$ returned values of $\mu_{\min }$ (ECM) larger than those returned by $\mu_{\min }$ (Young) and $\mu_{\min }$ (Algo. 5). The same situation occurs with the classical two-steps compared with both Young based approach and the new two-steps method (Algorithm 5). On the other hand, $\mu_{\min }$ (Young) is larger than $\mu_{\min }($ Algo. 5) in $94 \%$ cases. Noticing that since both Young based approach and NTSM are based on the gridding method, it is difficult to provide exact comparison. Thus, taking into consideration the fact that we have fixed $\alpha_{1}=\alpha_{2}=1$ and $\epsilon_{4}=0.0101$, the obtained results in this particular case show that $\mu_{\min }$ (Young) is very close to $\mu_{\min }$ (Algo.5) in $100 \%$ cases. All the conclusions are summarized in Figure 2 . We can see that Algorithm 5 performs better than the Young inequality based approach, the equality constraint based approach and the classical two-steps method.

\section{CONCLUSION}

The proposed design method for observer-based state feedback developed in this paper provides less conservative LMI conditions than those established in the literature. Indeed, the new design algorithm works in two-steps, but the procedure is completely different than the classical two-steps method. The general algorithm combining the Young's relation based technique and the new two-steps algorithm is particularly interesting. 


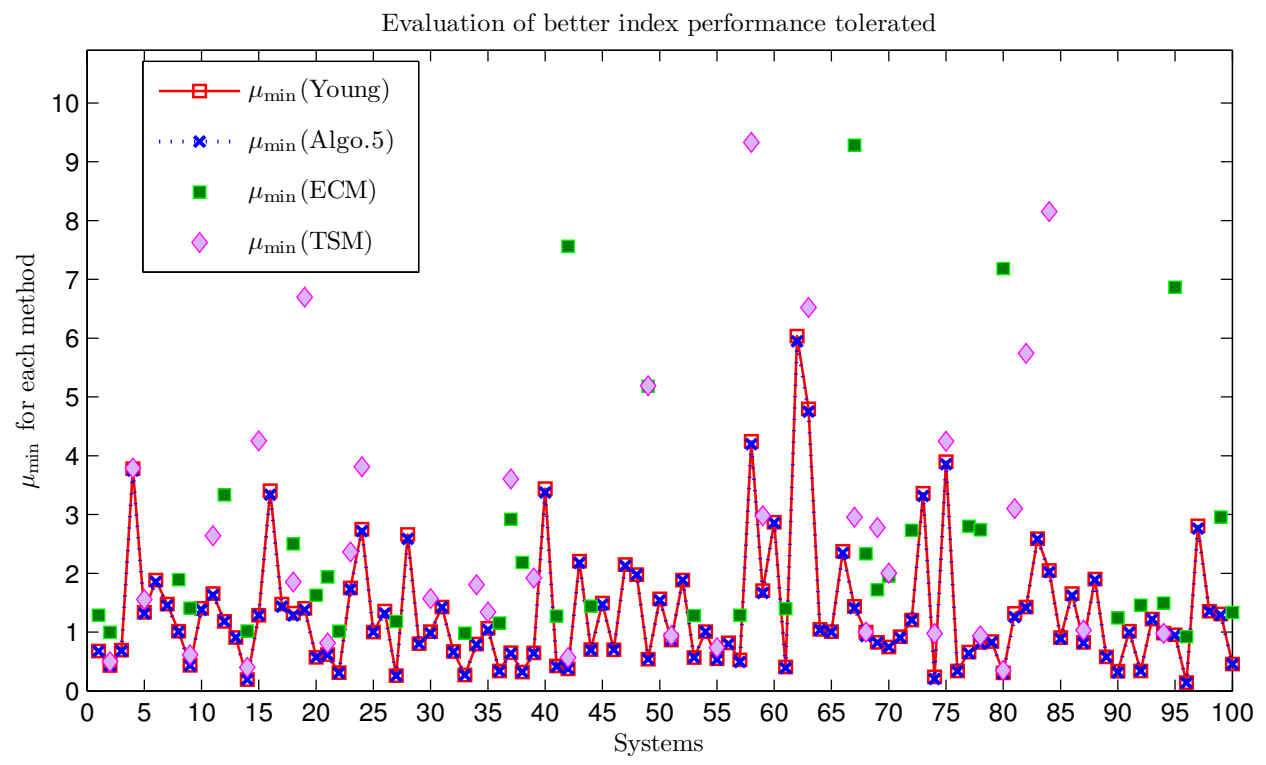

Figure 2. Evaluation of $\mu_{\min }$ for each method

Indeed, this general algorithm contains additional degrees of freedom and encompasses both Lien's method and the Young's relation based approach. The analytical and numerical comparisons show clearly the superiority of the proposed methodology. In future work, we hope to extend our technique to switching systems with unknown switching modes and to linear parameter varying systems with inexact parameters. Indeed, these classes of systems are particularly difficult from the observer-based stabilization point of view.

\section{REFERENCES}

1. M. Arcak and P. Kokotovic. Observer-based control of systems with slope-restricted nonlinearities. IEEE Transactions on Automatic Control, 46(7):1146-1150, 2001.

2. A.N. Atassi and H.K. Khalil. Separation results for the stabilization of nonlinear systems using different high-gain observer designs. Systems \& Control Letters, 39(3):183-191, 2000.

3. Stephen Boyd, Laurent El Ghaoui, Eric Feron, and Venkataramanan Balakrishnan. Linear matrix inequalities in system and control theory, volume 15 of SIAM Studies in Applied Mathematics. Society for Industrial and Applied Mathematics (SIAM), Philadelphia, PA, 1994.

4. Huijun Gao, Changhong Wang, and Lu Zhao. Comments on: ”An LMI-based approach for robust stabilization of uncertain stochastic systems with time-varying delays”. IEEE Transactions on Automatic Control, 48(11):2073-2074, 2003.

5. H.F. Grip, A. Saberi, and T.A. Johansen. Observers for interconnected nonlinear and linear systems. Automatica, 48(7):1339$1346,2012$. 
6. W.P.M.H. Heemels, J. Daafouz, and G. Millerioux. Observer-based control of discrete-time LPV systems with uncertain parameters. IEEE Transactions on Automatic Control, 55(9):2130-2135, 2010.

7. E. Hendricks and J.B. Luther. Model and observer based control of internal combustion engines. In Proceedings of the International Workshop on Modeling, Emissions and Control in Automotive Engines (MECA), Fisciano, Italy, pages 9-21, 2001.

8. S. Ibrir. Design of static and dynamic output feedback controllers through Euler approximate models: uncertain systems with norm-bounded uncertainties. IMA J. Math. Control Inform., 25(3):281-296, 2008.

9. S. Ibrir. Static output feedback and guaranteed cost control of a class of discrete-time nonlinear systems with partial state measurements. Nonlinear Analysis, 68(7):1784-1792, 2008.

10. S. Ibrir and S. Diopt. Novel LMI conditions for observer-based stabilization of Lipschitzian nonlinear systems and uncertain linear systems in discrete-time. Applied Mathematics and Computation, 206(2):579-588, 2008.

11. S. Ibrir, W.F. Xie, and C.Y. Su. Observer-based control of discrete-time Lipschitzian non-linear systems: application to one-link flexible joint robot. International Journal of Control, 78(6):385-395, 2005.

12. L. Jetto and V. Orsini. Efficient LMI-based quadratic stabilization of interval LPV systems with noisy parameter measures. IEEE Transactions on Automatic Control, 55(4):993-998, 2010.

13. I. Karafyllis and C. Kravaris. Robust output feedback stabilization and nonlinear observer design. Systems \& control letters, 54(10):925-938, 2005 .

14. H. Kheloufi, A. Zemouche, F. Bedouhene, and M. Boutayeb. A new observer-based stabilization method for linear systems with uncertain parameters. In 12th European Control Conference, ECC'13, Zurich, Switzerland, 2013.

15. H. Kheloufi, A. Zemouche, F. Bedouhene, and M. Boutayeb. On LMI conditions to design observer-based controllers for linear systems with parameter uncertainties. Automatica, 49(12):3700-3704, 2013.

16. H. Kheloufi, A. Zemouche, F. Bedouhene, and H. Souley-Ali. A robust $\mathcal{H}_{\infty}$ observer-based stabilization method for systems with uncertain parameters and Lipschitz nonlinearities. International Journal of Robust and Nonlinear Control, 26(9):19621979, 2016.

17. H. Lens. Fast robust stabilization by saturating output feedback of uncertain linear systems with input constraints. 48th IEEE Conference on Decision and Control and 28th Chinese Control Conference, Shanghai, P.R. China, December 2009.

18. C.H. Lien. Robust observer-based control of systems with state perturbations via LMI approach. IEEE Transactions on Automatic Control, 49(8):1365-1370, 2004.

19. E. Ostertag. An improved path-following method for mixed $H_{2} / H_{\infty}$ controller design. IEEE Transactions on Automatic Control, 53(8):1967-1971, 2008.

20. P.R. Pagilla, E.O. King, L.H. Dreinhoefer, and S.S. Garimella. Robust observer-based control of an aluminum strip processing line. EEE Transactions on Industry Applications, 36(3):865-870, 2000.

21. C. Scherer, P. Gahinet, and M. Chilali. Multiobjective output-feedback control via lmi optimization. IEEE Transactions on Automatic Control, 42(7):896-911, 1997.

22. B. Song and J.K. Hedrick. Observer-based dynamic surface control for a class of nonlinear systems: an LMI approach. IEEE Transactions on Automatic Control, 49(11):1995-2001, 2004. 
23. S.S. Stankovic, D.M. Stipanovic, and D.D. Siljak. Decentralized dynamic output feedback for robust stabilization of a class of nonlinear interconnected systems. Automatica, 43(5):861-867, 2007.

24. A. Swarnakar, H.J. Marquez, and T. Chen. A new scheme on robust observer-based control design for interconnected systems with application to an industrial utility boiler. IEEE Transactions on Control Systems Technology, 16(3):539-547, 2008.

25. O. Toker and H. Osbay. On the NP-hardness of solving bilinear matrix inequalities and simultaneous stabilization with static output feedback. IEEE American Control Conference, Seattle, WA, USA, Jun 1995.

26. S. Wang and Y. Jiang. Comment on "On LMI conditions to design observer-based controllers for linear systems with parameter uncertainties [Automatica 49 (2013) 3700-3704]”. Automatica, 50(10):2732-2733, 2014.

27. A. Zemouche and M. Boutayeb. On LMI conditions to design observers for Lipschitz nonlinear systems. Automatica, 49(2):585591, 2013.

28. A. Zemouche, R. Rajamani, B. Boulkroune, H. Rafaralahy, and M. Zasadzinski. Convex optimization based dual gain observer design for Lipschitz nonlinear systems. In IEEE American Control Conference, Boston, MA, USA., July 2016.

29. A. Zemouche, R. Rajamani, B. Boulkroune, H. Rafaralahy, and M. Zasadzinski. $\mathcal{H}_{\infty}$ circle criterion observer design for Lipschitz nonlinear systems with enhanced LMI conditions. In IEEE American Control Conference, Boston, MA, USA., July 2016. 